\title{
SUPERSIMPLE STRUCTURES WITH A DENSE INDEPENDENT SUBSET
}

\author{
ALEXANDER BERENSTEIN, JUAN FELIPE CARMONA, AND EVGUENI VASSILIEV
}

\begin{abstract}
Based on the work done in 6, 11 in the o-minimal and geometric settings, we study expansions of models of a supersimple theory with a new predicate distiguishing a set of forking-independent elements that is dense inside a partial type $\mathcal{G}(x)$, which we call $H$-structures. We show that any two such expansions have the same theory and that under some technical conditions, the saturated models of this common theory are again $H$-structures. We prove that under these assumptions the expansion is supersimple and characterize forking and canonical bases of types in the expansion. We also analyze the effect these expansions have on one-basedness and CM-triviality. In the one-based case, when $T$ has $S U$-rank $\omega^{\alpha}$ and the $S U$-rank is continuous, we take $\mathcal{G}(x)$ to be the type of elements of $S U$-rank $\omega^{\alpha}$ and we describe a natural "geometry of generics modulo $H$ " associated with such expansions and show it is modular.
\end{abstract}

\section{INTRODUCTION}

There are several papers that deal with expansions of simple theories with a new unary predicate. For example, there is the expansion with a random subset [10] that gives a case where the new theory is again simple and forking remains the same, in contrast to the case of lovely pairs [2, 20, where the pair is usually much richer and the complexity of forking is related to the geometric properties of the underlying theory [20].

In [6] the first and the third authors studied, in the setting of geometric structures, adding a predicate for an algebraically independent set $H$ which is dense and codense in a model $M$ (meaning every non-algebraic formula in a single variable has a realization in $H$ and a realization not algebraic over $H$ and its parameters). Such expansions are called $H$-structures and came as a generalization of ideas developed in the framework of o-minimal theories in [11]. The key tool used in [6] was that the closure operator acl has the exchange property and thus gives a matroid that interacts well with the definable subsets. A special case under consideration was SU-rank one theories, where forking independence agrees with algebraic independence. In the $S U$-rank one setting the authors characterized forking in the expansion and gave a description of canonical bases. As in the lovely pair case, the complexity of forking is related to the underlying geometry of the base theory $T$.

2000 Mathematics Subject Classification. 03C45.

Key words and phrases. supersimple theories, pregeometries, unary predicate expansions, onebasedness, ampleness, CM-triviality .

The first author was partially supported by the project of Colciencias "Métodos de estabilidad en clases no estables". The authors would like to thank Daniel Palacín for some helpful coversations and the referee for some valuable suggestions. 
In this paper we bring together ideas from lovely pairs of simple theories and $H$-structures to the setting of supersimple theories, we use as a main tool forking independence. We fix a complete supersimple theory and a type $\mathcal{G}(x)$ and consider the expansion where we name as a predicate realizations of $\mathcal{G}(x)$ that are forking independent and that satisfy a density property with respect to $\mathcal{G}(x)$, namely every formula that belongs to a non-forking extension of $\mathcal{G}(x)$ has a realization in the predicate. We also assume that the structure is rich with respect to the predicate: every formula $\varphi(x, \vec{a})$ has a realization that is forking independent from the predicate over $\vec{a}$.

We call such expansions $H$-structures associated to $\mathcal{G}$. We first prove that all such structures define the same theory, which we call $T_{\mathcal{G}}^{\text {ind }}$. When a $|T|^{+}$-saturated model of $T_{\mathcal{G}}^{\text {ind }}$ is again an $H$-structure associated to $\mathcal{G}$ we say that the class of $H$-structures associated to $\mathcal{G}$ is first order. We show that the class is first order under a weakening of wnfcp and under some definability condition on non-forking extensions of $\mathcal{G}(x)$. When the class if first order, we prove that the extension is supersimple and characterize forking. In particular, we get a clear description of canonical bases in the expansion, up to interalgebraicity (see Proposition [5.5).

Assume now that $T$ is a one-based supersimple theory of SU-rank $\omega^{\alpha}$ and that the $S U$-rank is continuous. Take $\mathcal{G}(x)$ to be the (partial) type of rank $\omega^{\alpha}$, which can be seen as the generic elements in $T$. Then there is a natural pregeometry associated to the generics, namely $a \in \operatorname{cl}(B)$ if $S U(a / B)<\omega^{\alpha}$. We then use this expansion to study the underlying geometry of the closure operator localized in $H$. We show that if $T$ is a one-based supersimple theory of SU-rank $\omega^{\alpha},(N, H)$ a sufficiently (e.g. $|T|^{+}-$) saturated $H$-structure, then the localized closure operator $\operatorname{cl}(-\cup H)$ is modular and its associated geometry is a disjoint union of projective geometries over division rings and trivial geometries. These closures have been studied also for lovely pair like constructions, see for example the work of Fornasiero on lovely pairs of closure operators [12].

Of special interest is the effect of our expansion on the geometric complexity, namely the ampleness hierarchy. Following the ideas of [8], we show that the expansion preserves CM-triviality. When $T$ is one-based, $\mathcal{G}$ is $x=x$, then $T_{\mathcal{G}}^{\text {ind }}$ is one-based if and only if forking is trivial.

When $T$ is one-based of SU-rank $\omega^{\alpha}$ with continuous rank and we choose $\mathcal{G}$ to be the partial type of SU-rank $\omega^{\alpha}$, then, as described above, the theory induces a closure operator: $a \in \operatorname{cl}(B)$ if $S U(a / B)<\omega^{\alpha}$. In this setting, $T_{\mathcal{G}}^{i n d}$ is one-based if and only if the pregeometry associated to $\mathrm{cl}$ is trivial.

Finally we mention another paper related to this expansion. Assume the theory $T$ is superstable and $\mathcal{G}(x)$ is a stationary type over $\emptyset$, then the interpretation of the predicate is a Morley sequence; this case is related to the work done in [1].

This paper is organized as follows. In section 2 we start with a complete supersimple theory $T$ and and a type-definable set $\mathcal{G}$ and we define the class of $H$ structures associated to a theory $\mathcal{G}$. We show that two such $H$-structures are elementarily equivalent and call $T_{\mathcal{G}}^{\text {ind }}$ this common theory. Finally we prove that under some technical conditions (elimination of the quantifier $\exists^{\text {large }}$ and the type definability of the predicates $\left.Q_{\varphi, \psi}\right)$ the saturated models of $T_{\mathcal{G}}^{\text {ind }}$ are again $H$ structures.

In section 3 we study several examples of supersimple theories: $S U$-rank one structures, differentially closed fields, the free pseudoplane, vector spaces with a 
generic automorphism, $\omega$-stable theories with definable Morley rank, $H$-pairs and lovely pairs of geometric theories. In most cases we show the corresponding theory of $H$-structures with a reasonable choice for $\mathcal{G}$ is first order. In the cases of ACFA and a vector space with a generic automorphism, when $\mathcal{G}$ is the generic type, being first order is reduced to type definability of the predicates $Q_{\varphi, \psi}$.

In section 4 we analyze the definable sets in the expansion, we prove that every definable set is a boolean combination of old formulas bounded by existential quantifiers over the new predicate. In section 5 we characterize forking in the expansion and characterize canonical bases. In section 6 we study the question of preservation of one-basedness and then in section 7 the preservation of CM-triviality under our expansion. Finally in section 8 we consider the special case where $T$ is a one-based supersimple theory of SU-rank $\omega^{\alpha}$ such that the $S U$-rank is continuous and take $\mathcal{G}(x)$ to be the (partial) type of rank $\omega^{\alpha}$. We study the geometry of $\operatorname{cl}(-\cup H)$, where $\mathrm{cl}$ is the closure associated to the generic elements in the theory.

\section{2. $H$-STRUCTURES: DEFINITION AND FIRST PROPERTIES}

Let $T$ be a supersimple theory in a language $\mathcal{L}$, let $M \models T$ be a monster model. Since our only assumption on $T$ is supersimplicity, if needed, we can assume $T=$ $T^{e q}$. Fix a partial 1-type $\mathcal{G}(x)$ over $\emptyset$.

For $M \models T, A \subset M$ and $b \in M$, we say that that $b$ is large over $A$ (or that $\operatorname{tp}(b / A)$ is large $)$ if $\operatorname{tp}(b / A)$ is a non-forking extension of $\mathcal{G}$. Otherwise we say that $b$ is small over $A$ (or that $\operatorname{tp}(b / A)$ is small).

We will say a formula $\varphi(x, \vec{a})$ is large if there is $b \models \varphi(x, \vec{a})$ such that $b$ is large over $\vec{a}$. We will say a formula $\varphi(x, \vec{a})$ is small if it is not large. That is, for all $b=\varphi(x, \vec{a}), \operatorname{tp}(b / \vec{a})$ either does not extend $\mathcal{G}$ or it is a forking extension of $\mathcal{G}$.

The goal of this paper is to merge the ideas on $H$-structures developed in [4 with those of lovely pairs of simple theories [2] and develop the notion of $H$-structures relative to the family $\mathcal{G}$.

Following the ideas from [4, we let $H$ be a new unary predicate that does not appear in $\mathcal{L}$ and define $\mathcal{L}_{H}=\mathcal{L} \cup\{H\}$. So an $\mathcal{L}_{H}$-structure is a pair $(M, H(M))$, where $H(M) \subset M$.

Notation 2.1. Let $(M, H(M))$ be an $\mathcal{L}_{H}$-structure and let $A \subset M$. We write $H(A)$ for $H(M) \cap A$.

Notation 2.2. Throughout this paper independence means forking independence in the sense of $T$ and we use the familiar symbol $\downarrow$. We write $\operatorname{tp}(\vec{a})$ for the $\mathcal{L}$-type of $a$ and $\mathrm{dcl}$, acl for the definable closure and the algebraic closure in the language $\mathcal{L}$. Similarly we write $\mathrm{dcl}_{H}, \mathrm{acl}_{H}, \mathrm{tp}_{H}$ for the definable closure, the algebraic closure and the type in the language $\mathcal{L}_{H}$.

Definition 2.3. We say that $(M, H(M))$ is an $H$-structure associated to $\mathcal{G}$ if

(1) For every $h \in H(M), h \models \mathcal{G}$.

(2) For all $n \geq 1$, if $h_{1}, \ldots, h_{n} \in H(M)$ are distinct, then they are independent.

(3) (Density/coheir property) If $A \subset M$ is finite and $q \in S_{1}(A)$ is the type of a non-forking extension of $\mathcal{G}$, there is $a \in H(M)$ such that $a \models q$.

(4) (Co-density/extension property) If $A \subset M$ is finite and $q \in S_{1}(A)$, there is $a \in M, a \models q$ and $a \downarrow_{A} H(M)$.

Lemma 2.4. The pair $(M, H(M))$ is an $H$-structure associated to $\mathcal{G}$ if and only if: 
(1) $H(M) \models \mathcal{G}$.

(2) For all $n \geq 1$, if $h_{1}, \ldots, h_{n} \in H(M)$ are distinct, then they are independent.

(2') (Generalized density/coheir property) If $A \subset M$ is finite and $q \in S_{n}(A)$ is the type of an independent $n$-tuple of non-forking extensions of $\mathcal{G}$, then there is $\vec{a} \in H(M)^{n}$ such that $\vec{a} \models q$.

(3') (Generalized co-density/extension property) If $A \subset M$ is finite and $q \in$ $S_{n}(A)$, then there is $\vec{a} \in M^{n}$ realizing $q$ such that $\operatorname{tp}(\vec{a} / A \cup H(M))$ does not fork over $A$.

Proof. We prove (2') and leave (3') to the reader. Let $\vec{b} \models q$, we may write $\vec{b}=$ $\left(b_{1}, \ldots, b_{n}\right)$. Since $(M, H(M))$ is an $H$-structure, applying the density property we can find $a_{1} \in H(M)$ such that $\operatorname{tp}\left(a_{1} / A\right)=\operatorname{tp}\left(b_{1} / A\right)$. Let $q\left(x, b_{1}, A\right)=\operatorname{tp}\left(b_{2}, b_{1}, A\right)$ and let $A_{1}=A \cup\left\{a_{1}\right\}$. Now consider the type $q\left(x, a_{1}, A\right)$ over $A_{1}$, which is some non-forking extension of $\mathcal{G}$. Applying the density property we can find $a_{2} \in H(M)$ such that $\operatorname{tp}\left(a_{2}, a_{1} / A\right)=\operatorname{tp}\left(b_{2}, b_{1} / A\right)$. We continue inductively to find the desired tuple $\left(a_{1}, a_{2}, \ldots, a_{n}\right)$.

Note that if $(M, H(M))$ is an $H$-structure, the extension property implies that $M$ is $\aleph_{0}$-saturated. Also note that Definition 2.3 can be generalized to the setting of simple theories following the ideas of [2, Definition 3.1]. Finally note that if the codensity property holds for types in the real sort, then it also holds for types in imaginary sorts.

Definition 2.5. Let $A$ be a subset of an $H$-structure $(M, H(M))$. We say that $A$ is $H$-independent if $A$ is independent from $H(M)$ over $H(A)$.

Lemma 2.6. Any model $M$ of $T$ with a distinguished independent subset $H(M)$ can be embedded in an $H$-structure in an $H$-independent way.

Proof. Given any model $M$ with a distinguished independent subset $H(M)$ of elements realizing $\mathcal{G}$, we can always find an elementary extension $N$ of $M$ and a set $H(N)$ of independent realizations of $\mathcal{G}$ extending $H(M)$ such that for every generic 1-type $p(x, \operatorname{acl}(\vec{m}))$ (i.e. $p(x, \operatorname{acl}(\vec{m}))$ is a non-forking extension of $\mathcal{G}$ ), where $\vec{m} \in M$, there is $d \in H(N)$ such that $d \models p(x, \operatorname{acl}(\vec{m}))$ and $d \downarrow_{H(M)} \vec{m}$. Add a similar statement for the extension property. Now apply a chain argument.

In particular, $H$-structures exist. The following is the main result from this section:

Proposition 2.7. Let $(M, H)$ and $(N, H)$ be sufficiently saturated $H$-structures, $\vec{a} \in M$ and $\vec{a}^{\prime} \in N H$-independent tuples such that $\operatorname{tp}(\vec{a}, H(\vec{a}))=\operatorname{tp}\left(\vec{a}^{\prime}, H\left(\vec{a}^{\prime}\right)\right)$. Then $\operatorname{tp}_{H}(\vec{a})=\operatorname{tp}_{H}\left(\vec{a}^{\prime}\right)$.

Proof. Write $\vec{a}=\vec{a}_{0} \vec{a}_{1} \vec{h}$, where $\vec{a}_{0}$ is independent over $H(M), \vec{h}=H(\vec{a}) \in H(M)$ and $\vec{a}_{1}$ is small over $\vec{a}_{0} \vec{h}$. Similarly write $\vec{a}^{\prime}=\vec{a}_{0}^{\prime} \vec{a}_{1}^{\prime} \vec{h}^{\prime}$.

It suffices to show that for any $b \in M$ there are $\vec{h}_{1} \in H(M), \vec{h}_{1}^{\prime} \in H(N)$ and $b^{\prime} \in N$ such that $\vec{a} \vec{h}_{1} b$ and $\vec{a}^{\prime} \vec{h}_{1}^{\prime} b^{\prime}$ are each $H$-independent, $\operatorname{tp}\left(\vec{a}_{0} \vec{a}_{1} \vec{h} \vec{h}_{1} b\right)=$ $\operatorname{tp}\left(\vec{a}_{0}^{\prime} \vec{a}_{1}^{\prime} \vec{h}^{\prime} \vec{h}_{1}^{\prime} b^{\prime}\right)$, and $b \in H(M)$ iff $b^{\prime} \in H(N)$.

Case 1: $b \in H(M), b$ is small over $\vec{a}$. Then $\operatorname{tp}(b / \vec{a})$ forks over $\emptyset$. Since $\vec{a}$ is $H$-independent, we must have $b \downarrow_{\vec{h}} \vec{h} \vec{a}$ and by transitivity $b\lfloor\vec{h}$. Since $H$ forms an independent set we must have $b \in \vec{h}$. Let $b^{\prime} \in \vec{h}^{\prime}$ be such that $\operatorname{tp}\left(b^{\prime} \vec{a}^{\prime}\right)=\operatorname{tp}(b \vec{a})$ and the result follows. Here we can take $\vec{h}_{1}$ and $\vec{h}_{1}^{\prime}$ to be empty. 
Case 2: $b \in H(M)$ and is large over $\vec{a}$. Then $t p(b / \vec{a})$ is generic, that is, it is a non-forking extension of $\mathcal{G}$. Let $f: M \rightarrow M^{\prime}$ be a partial elementary map sending $\vec{a}$ to $\vec{a}^{\prime}$. Then $p\left(x, \vec{a}^{\prime}\right)=f(t p(b / \vec{a}))$ is a non-forking extension of $\mathcal{G}$. By the density property, we can find $b^{\prime} \in H(N)$ such that $b^{\prime} \models p\left(x, \vec{a}^{\prime}\right)$. That is, $\operatorname{tp}\left(b^{\prime} \vec{a}^{\prime}\right)=\operatorname{tp}(b \vec{a})$. Note that both tuples $b \vec{a}, b^{\prime} \vec{a}^{\prime}$ are $H$-independent. Here again we can take $\vec{h}_{1}$ and $\vec{h}_{1}^{\prime}$ to be empty.

Case 3: $b \notin H(M)$. By supersimplicity, there is a finite tuple $\vec{h}_{1} \in H(M)$ such that $b \downarrow_{\vec{a} \vec{h}_{1}} H(M)$. We may assume that $\vec{h}_{1}$ is disjoint from $\vec{h}$ and thus independent from $\vec{h}$. Now observe that since $\vec{a}$ is $H$-independent, so is $\vec{a} \vec{h}_{1}$. By Case 2 , there is $\vec{h}_{1}^{\prime} \in H(N)$ such that $\operatorname{tp}\left(\vec{a} \vec{h}_{1}\right)=\operatorname{tp}\left(\vec{a}^{\prime} \vec{h}_{1}^{\prime}\right)$. Let $p\left(x, \vec{a} \vec{h}_{1}\right)=\operatorname{tp}\left(b / \vec{a} \vec{h}_{1}\right)$. Now use the extension property to find $b^{\prime} \in N$ such that $b^{\prime} \models p\left(x, \vec{a}^{\prime}, \vec{h}_{1}^{\prime}\right), b^{\prime} \downarrow_{\vec{a}^{\prime} \vec{h}_{1}^{\prime}} H(N)$. Then by transitivity $\vec{a}^{\prime} \vec{h}_{1}^{\prime} b^{\prime}$ is $H$-independent.

The previous result has the following consequence:

Corollary 2.8. All $H$-structures associated to $\mathcal{G}(x)$ are elementarily equivalent. $T$.

We write $T_{\mathcal{G}}^{i n d}$ for the common complete theory of all $H$-structures of models of

Definition 2.9. We say that $T_{\mathcal{G}}^{\text {ind }}$ is first order if the $|T|^{+}$-saturated models of $T_{\mathcal{G}}^{\text {ind }}$ are again $H$-structures associated to $\mathcal{G}$.

To axiomatize $T_{\mathcal{G}}^{i n d}$ and to show that $T_{\mathcal{G}}^{i n d}$ is first order, we follow the ideas of [20. Prop 2.15], 4] and [2. We will need several technical assumptions for this result

Definition 2.10. We say that being large is definable if for every formula $\varphi(x, \vec{y})$ there is a formula $\psi(\vec{y})$ such that for any $\vec{a} \in M, \varphi(x, \vec{a})$ is large if and only if $\psi(\vec{a})$. When this is the case, we write $\exists^{\text {large }} x \varphi(x, \vec{y})$ if $\psi(\vec{y})$ holds.

We also need the following definition from [2, Definition 2.4]:

Definition 2.11. Let $\psi(\vec{y}, \vec{z})$ and $\varphi(\vec{x}, \vec{y})$ be $\mathcal{L}$-formulas. $Q_{\varphi, \psi}$ is the predicate which is defined to hold of a tuple $\vec{c}($ in $M$ ) if for all $\vec{b}$ satisfying $\psi(\vec{y}, \vec{c})$, the formula $\varphi(\vec{x}, \vec{b})$ does not divide over $\vec{c}$.

The following result follows word by word from the proof of [2, Proposition 4.5], changing the elementary substructure for the predicate $H$ :

Proposition 2.12. The following are equivalent:

(1) $Q_{\varphi, \psi}$ is type-definable (in $M$ ) for all $\mathcal{L}$-formulas $\varphi(\vec{x}, \vec{y}), \psi(\vec{y}, \vec{z})$.

(2) Any $|T|^{+}$-saturated model of $T_{\mathcal{G}}^{\text {ind }}$ satisfies the extension property.

In 2] it has been proved that, whenever $T$ is a simple theory, its associated theory of lovely pairs $T_{P}$ is axiomatizable if and only if $T$ is low and the predicates $Q_{\varphi, \psi}$ are type definable. In this case we say that $T$ has the weak non-finite cover property (wnfcp) (For $T$ stable, it is known that $T_{P}$ is axiomatizable if and only if $T$ has the nfcp, therefore this definition is rather natural).

Corollary 2.13. Let $T$ be a supersimple theory that satisfies wnfcp. Then the extension property is first order. 
Finally we prove that under some strong assumptions, $T_{\mathcal{G}}^{\text {ind }}$ is first order:

Proposition 2.14. Assume $T$ eliminates $\exists^{\text {large }}$ and that the predicates $Q_{\varphi, \psi}$ are $\mathcal{L}$-type-definable for all $\mathcal{L}$-formulas $\varphi(\vec{x}, \vec{y}), \psi(\vec{y}, \vec{z})$. Then, if $(M, H) \models T_{\mathcal{G}}^{\text {ind }}$ is $|T|^{+}$-saturated then it is an $H$-structure associated to $\mathcal{G}$.

Proof. We start by showing that all elements in $H$ satisfy the partial type $\mathcal{G}$. Let $\theta(x) \in \mathcal{G}$, then $\forall x(H(x) \rightarrow \theta(x)) \in T_{\mathcal{G}}^{i n d}$. Thus for $h \in H(M), M \models \theta(h)$ and we have $H(M) \subset \mathcal{G}(M)$.

Let us prove that $(M, H)$ satisfies the density property. Let $p(x, \vec{a})$ be non-forking extension of $\mathcal{G}$. Let $\varphi(x, \vec{a}) \in p(x, \vec{a})$, so $\varphi(x, \vec{a})$ is large. Then $M \models \exists^{\text {large }} x \varphi(x, \vec{a})$ and all $H$-structures $(N, H)$ satisfy

$$
\forall \vec{y}\left(\exists^{\text {large }} x \varphi(x, \vec{y}) \rightarrow \exists h(H(h) \wedge \varphi(h, \vec{y})) .\right.
$$

Thus there is $h \in H(M)$ such that $M \models \varphi(h, \vec{a})$. Since $M$ is $|T|^{+}$-saturated, there is $h \in H(M)$ such that $M \models p(h, \vec{a})$.

Now we prove that the elements in $H$ are independent. Notice that the following sentence is true in every $H$-structure:

$$
\forall h_{0}, h_{1}, \ldots, h_{n} \in H\left(\left(\bigwedge h_{i} \neq h_{j}\right) \wedge \theta\left(h_{0}, h_{1}, \ldots, h_{n}\right) \rightarrow \exists^{\text {large }} x \theta\left(x, h_{1}, \ldots, h_{n}\right)\right) .
$$

Therefore if $d, c_{1}, \ldots, c_{n} \in H(M)$ are all distinct and $\theta\left(x, c_{1}, \ldots, c_{n}\right) \in \operatorname{tp}\left(d / c_{1}, \ldots, c_{n}\right)$ we have $\exists^{\text {large }} x \theta\left(x, c_{1}, \ldots, c_{n}\right)$ and in particular $\theta\left(x, c_{1}, \ldots, c_{n}\right)$ does not fork over $\emptyset$. Thus all formulas in the type $t p\left(d / c_{1}, \ldots, c_{n}\right)$ do not fork over $\emptyset$ and thus $d \downarrow\left\{c_{1}, \ldots, c_{n}\right\}$.

Finally by Proposition 2.12 any $|T|^{+}$-saturated model of $T_{\mathcal{G}}^{\text {ind }}$ satisfies the extension property.

\section{EXAmples}

In this section we give a list of examples of supersimple theories with a type definable set $\mathcal{G}$ that eliminate $\exists^{\text {large }}$ and where the extension property is first order. We also list some examples that eliminate the quantifier $\exists^{\text {large }}$ but where it remains as an open question if the extension property is first order.

3.1. Theories of $S U$-rank one. In this example we show that the results from [4] are a special case of our new setting. Our presentation will be sketchy, for more details see [4.

Let $T$ be a complete theory of $S U$-rank one and let $\mathcal{G}$ be the intersection of all the non-algebraic types (i.e. the set of all formulas whose negation is algebraic). Then $T$ is geometric, i.e. the algebraic closure satisfies the exchange property and $T$ eliminates $\exists^{\infty}$. In this setting a formula is large if it is non-algebraic and it is small if it is algebraic. Since $T$ eliminates $\exists^{\infty}$ then $T$ eliminates $\exists^{\text {large }}$. Finally since independence is captured by algebraic independence, then the extension property is first order.

3.2. Differentially closed fields. Let $T=D C F_{0}$, the theory of differentially closed fields. This theory is stable of $U$-rank $\omega$ and also $R M\left(D C F_{0}\right)=\omega$.

First we study the extension property. Recall that $D C F_{0}$ has quantifier elimination [14, Theorem 2.4] and eliminates imaginaries [14, Theorem 3.7]. It is proved in [14, Theorem 2.13] that $D C F_{0}$ has uniform bounding (i.e. it eliminates $\exists^{\infty}$ ) and 
thus it has nfcp. This is also explicitly explained in [14, page 52]. It follows by Corollary 2.13 that the extension property is first order.

Let $p(x)$ be the unique generic type of the theory, that is, the type of a differential trascendental. This type is complete, stationary and definable over $\emptyset$. Choose $\mathcal{G}$ to be the type $p(x)$. Let $\varphi(x, \vec{y})$ be a formula and let $\psi(\vec{y})$ be its $p$-definition. Then for $(K, d) \models D C F_{0}, \vec{a} \in K$, the formula $\varphi(x, \vec{a})$ is large iff $\psi(\vec{a})$. Thus this theory eliminates the quantifier $\exists^{\text {large }}$.

Also note that we can choose other partial types for $\mathcal{G}$. For example, let $\mathcal{G}$ be the non-algebraic constants, given by the type-definable set $\delta(x)=0, x \notin \operatorname{acl}(\emptyset)$. It is proved in [14, Lemma 5.6] that the only definable subsets of the field of constants are definable in the field language within the field of constants, thus the constants are stably embedded and strongly minimal. The partial type $\mathcal{G}$ corresponds to the generic type of $\delta(x)=0$, which is stationary and definable over $\emptyset$. As before, the definability of the type implies elimination of $\exists^{\text {large }}$.

Since beautifull pairs associated to nfcp theories are again nfcp (see [19]), we can also work on a lovely pair of differentially closed fields (which has $S U$-rank $\omega^{2}$ ) and choose $\mathcal{G}$ to be some stationary type in the pair definable over $\emptyset$, for example the type of the generic elements in the pair.

3.3. Free pseudoplane-infinite branching tree. Let $T$ be the theory of the free pseudoplane, that is, a graph without cycles such that every vertex has infinitely many edges. The theory of the free pseudoplane is stable of $U$-rank $\omega$ and $M R(T)=\omega$. For every $A, \operatorname{acl}(A)=\operatorname{dcl}(A)=A \cup\{x \mid$ there are points $a, b \in$ $A$ and a path connecting them passing trough $x\}$. For $A$ algebraically closed and $a$ a single element, $U(a / A)=d(a, A)$ where $d(a, A)$ is the minimum length of a path from $a$ to an element of $A$ or $\omega$ if there is no path; in this last case we say that $a$ is at infinite distance to $A$ or that $a$ is not connected to $A$. Note that there is a unique generic type over $A$, namely the type of an element which is not connected to $A$. Choose $\mathcal{G}(x)$ to be this type. The generic type is definable over $\emptyset$ and thus by definability of types $T$ eliminates the quantifier $\exists^{\text {large }}$.

An $H$-structure $(M, H)$ associated to $\mathcal{G}$ is an infinite collection of trees with an infinite collection of selected points $H(M)$ at infinite distance one from the other and with infinite many trees not connected to them. If $(N, H) \models T h(M, H)$, then $N$ has infinitely many selected points $H(N)$ at infinite distance one from the other.

If $(N, H)$ is $\aleph_{0}$-saturated, then by saturation it also has infinitely many trees which are not connected to the points $H(N)$. We will prove that in this case $(N, H)$ is an $H$-structure associated to $\mathcal{G}(x)$. Since $T$ eliminates the quantifier $\exists^{\text {large }}$, the density property holds as well as the fact that the elements in $H(N)$ are independent (both these properties are easy to check directly too). Now let $A \subset N$ be finite and assume that $A=\operatorname{dcl}(A)$ and let $c \in N$. If $U(c / A)=\omega$ choose a point $b$ in a tree not connected to $A \cup H$, then $\operatorname{tp}(c / A)=\operatorname{tp}(b / A)$ and $b \downarrow_{A} H$. If $U(c / A)=0$ there is nothing to prove. If $U(c / A)=n>0$, let $a$ be the nearest point from $A$ to $c$. Since there is at most one point of $H$ connected to $a$ and the trees are infinitely branching, we can choose a point $b$ with $d(b, a)=n$ and such that $d(b, A \cup H)=n$; then $\operatorname{tp}(c / A)=\operatorname{tp}(b / A)$ and $b \downarrow_{A} H$. This proves that $(N, H)$ is an $H$-structure and that that $T_{\mathcal{G}}^{\text {ind }}$ is first order.

3.4. Theories of Morley rank omega with definable Morley rank. Let $T$ be a $\omega$-stable theory of rank $\omega$ and let $M \models T$ be $|T|^{+}$-saturated. Assume also that 
the Morley rank is definable, that is, for every formula $\varphi(x, \vec{y})$ without parameters and every $\alpha \in\{0,1, \ldots, \omega\}$ there is a formula $\psi_{\alpha}(\vec{y})$ without parameters such that for $\vec{a} \in M, M R(\varphi(x, \vec{a})) \geq \alpha$ if and only if $\psi_{\alpha}(\vec{a})$. To simplify the notation, we will write $\operatorname{MR}(\varphi(x, \vec{a})) \geq \alpha$ instead of $\psi_{\alpha}(\vec{a})$. Let $\mathcal{G}$ be the intersection of the types of Morley rank $\omega$, i.e. the formulas whose complement have rank $<\omega$. We will prove that $T_{\mathcal{G}}^{\text {ind }}$ is first order.

Elimination of $\exists^{\text {large }}$ : Consider first $\varphi(x, \vec{y})$ and let $\vec{b} \in M$. Then $\varphi(x, \vec{b})$ is large if and only if $\operatorname{MR}(\varphi(x, \vec{b})) \geq \omega$, so $T$ eliminates the quantifier $\exists^{\text {large }}$.

Extension property: Assume that $(M, H)$ is an $H$-structure associated to $\mathcal{G}$ and let $(N, H) \models T h(M, H)$ be $|T|^{+}$-saturated. Let $a \in N$ and let $\vec{b} \in N$. If $M R(\operatorname{tp}(a / \vec{b}))=0$ there is nothing to prove. Assume then that $M R(\operatorname{tp}(a / \vec{b}))=n>$ 0 .

Let $\varphi(x, \vec{y}) \in \operatorname{tp}(a, \vec{b})$ with $M R(\varphi(x, \vec{b}))=n$ and $M d(\varphi(x, \vec{b}))=M d(\operatorname{tp}(a / \vec{b}))$. Let $\left(a^{\prime}, \vec{b}^{\prime}\right) \models t p(a, \vec{b})$ belong to $M$. Since $(M, H)$ is an $H$-structure, we may assume that $a^{\prime} \downarrow_{\overrightarrow{b^{\prime}}} H$ and thus for every formula $\theta(x, \vec{y}, \vec{z})$ and every tuple $\vec{h} \in H$, if $M R\left(\theta\left(x, \vec{b}^{\prime}, \vec{h}\right)\right)<M R\left(\varphi\left(x, \vec{b}^{\prime}\right)\right)=n$ then $\neg \theta\left(x, \vec{b}^{\prime}, \vec{h}\right) \in \operatorname{tp}\left(a^{\prime} / \vec{b}^{\prime} H\right)$. So $(M, H) \models$ $\forall d^{\prime}\left(M R\left(\varphi\left(x, \vec{d}^{\prime}\right)\right) \geq n \rightarrow \exists c \varphi\left(c, \overrightarrow{d^{\prime}}\right) \wedge \forall \vec{h} \in H\left(M R\left(\theta\left(x, \overrightarrow{d^{\prime}}, \vec{h}\right)\right)<n \rightarrow \neg \theta\left(c, \overrightarrow{d^{\prime}}, \vec{h}\right)\right)\right.$.

Since $(N, H) \models T h(M, H)$ is $|T|^{+}$-saturated, we can find $a^{\prime}$ such that $M R\left(a^{\prime} / \vec{b}\right) \geq$ $n, \models \varphi\left(a^{\prime}, b\right)$ and whenever $\vec{h} \in H(N)$ and $\theta(x, \vec{b}, \vec{h})$ is a formula with Morley rank smaller than $n$ we have $\neg \theta\left(a^{\prime}, \vec{b}, \vec{h}\right)$. This shows that $\operatorname{MR}\left(a^{\prime} / \vec{b} H\right)=\operatorname{MR}\left(a^{\prime} / \vec{b}\right)=$ $M R(a / \vec{b}), M d\left(a^{\prime} / \vec{b}\right)=M d(a / \vec{b})$, both $a$ and $a^{\prime}$ are generics of the formula $\varphi(x, \vec{b})$ and thus $\operatorname{tp}(a / \vec{b})=\operatorname{tp}\left(a^{\prime} / \vec{b}\right)$. Finally by construction $a^{\prime} \downarrow_{\vec{b}} H$. It follows that $T_{\mathcal{G}}^{\text {ind }}$ is first order.

3.5. $H$-triples. Recall from 4 that if $T_{0}$ is supersimple $S U$-rank one theory, then $T=T_{0}^{\text {ind }}$ is supersimple (of rank 1 or $\omega$, depending on whether the geometry $T_{0}$ is trivial or not). The models of $T$ are structures of the form $\left(M, H_{1}\right)$, where $M \models T_{0}$ and $H_{1}$ is a acl ${ }_{0}$-dense and acl $0_{0}$-codense subset of $M$. We write $\mathcal{L}_{0}$ for the language associated to $T_{0}$ and $\mathcal{L}$ for the language associated to $T$. Similarly, we write acl for the algebraic closure in the language $\mathcal{L}_{0}$ and for $A \subset M \models T_{0}$, we write $S_{n}^{0}(A)$ for the space of $\mathcal{L}_{0}$ - $n$-types over $A$.

In this subsection we change our notation and we let $H_{2}$ be a new predicate symbol that will be interpreted by a dense and codense independent subset of $\left(M, H_{1}\right)$ (in the sense of forking independence in $T$ ).

The structures $\left(M, H_{1}, H_{2}\right)$ were already studied in 4 . We recall the definitions and the main result. The main tool for studying $T_{\mathcal{G}}^{i n d}$ is to take into account the base theory $T_{0}$ and use triples.

Definition 3.1. We say that $\left(M, H_{1}(M), H_{2}(M)\right)$ is an $H$-triple associated to $T_{0}$ if:

(1) $M \models T_{0}, H_{1}(M)$ is an $a c l_{0}$-independent subset of $M, H_{2}(M)$ is an $a c l_{0}$ independent subset of $M$ over $H_{1}$.

(2) (Density property for $H_{1}$ ) If $A \subset M$ is finite and $q \in S_{1}^{0}(A)$ is non-algebraic, there is $a \in H_{1}(M)$ such that $a \models q$.

(3) (Density property for $H_{2} / H_{1}$ ) If $A \subset M$ is finite and $q \in S_{1}^{0}(A)$ is nonalgebraic, there is $a \in H_{2}(M)$ such that $a \models q$ and $a \notin \operatorname{acl}_{0}\left(A \cup H_{1}(M)\right)$.

(4) (Extension property) If $A \subset M$ is finite and $q \in S_{1}^{0}(A)$ is non-algebraic, there is $a \in M, a \models q$ and $a \notin \operatorname{acl}_{0}\left(A \cup H_{1}(M) \cup H_{2}(M)\right)$. 
It is observed in [4 that if $\left(M, H_{1}(M), H_{2}(M)\right),\left(N, H_{1}(N), H_{2}(N)\right)$ are $H$ triples, then $T h\left(M, H_{1}(M), H_{2}(M)\right)=T h\left(N, H_{1}(N), H_{2}(N)\right)$ and we denote the common theory by $T_{0}^{\text {tri }}$.

We will now apply our construction to $T=T_{0}^{\text {ind }}$. Let $\mathcal{G}$ be the partial type in $T$ representing $M \backslash \operatorname{acl}\left(H_{1}(M)\right)$. Then an $H$-structure with respect to $\mathcal{G}$ will be given by $\left(M, H_{1}, H_{2}\right)$ where $H_{2}(M) \subset M \backslash \operatorname{acl}\left(H_{1}(M)\right), H_{2}(M)$ is forking-independent in the sense of $\left(M, H_{1}\right)$ (which is equivalent to acl $\left(-\cup H_{1}(M)\right)$-independence or, in the notation of [4], scl-independence), such that for any finite $A \subset M$ and $a \in M$ such that $a \notin \operatorname{acl}\left(A H_{1}(M)\right), \operatorname{tp}(a / A)$ (in the sense of $\left(M, H_{1}\right)$ ) is realized in $H_{2}(M)$ and in $M \backslash \operatorname{acl}\left(A H_{1}(M) H_{2}(M)\right)$. This agrees with the notion of scl-structure in [4.

The following result is proved (in the more general geometric setting) in 4] (Prop. 4.6).

Proposition 3.2. Let $T_{0}$ be an $S U$ rank 1 supersimple theory, let $M \models T_{0}$ and let $H_{1}(M) \subset M, H_{2}(M) \subset M$ be distinguished subsets. Then $\left(M, H_{1}(M), H_{2}(M)\right)$ is a scl-structure associated to $T_{0}$ if and only if $\left(M, H_{1}(M), H_{2}(M)\right)$ is an $H$-triple of the theory $T_{0}$.

Thus, to show that the class of $H_{2}$-structures associated to $T$ is first order, it suffices to prove that this is the case for $H$-triples associated to $T_{0}$. As pointed out in [4] we have:

Proposition 3.3. The theory $T^{t r i}$ is axiomatized by:

(1) $M \models T_{0}, H_{1}(M)$ is an acl $0_{0}$-independent subset of $M, H_{2}(M)$ is an aclindependent subset of $M$ over $H_{1}$.

(2) For all $\mathcal{L}_{0}$-formulas $\varphi(x, \vec{y})$ $\forall \vec{y}\left(\varphi(x, \vec{y})\right.$ nonalgebraic $\Longrightarrow \exists x\left(\varphi(x, \vec{y}) \wedge x \in H_{1}\right)$.

(3) For all $\mathcal{L}_{0}$-formulas $\varphi(x, \vec{y}), m \in \omega$, and all $\mathcal{L}_{0}$-formulas $\psi\left(x, z_{1}, \ldots, z_{m}, \vec{y}\right)$ such that for some $n \in \omega \forall \vec{z} \forall \vec{y} \exists \leq n x \psi(x, \vec{z}, \vec{y}) \quad($ so $\psi(x, \vec{y}, \vec{z})$ is always algebraic in $x)$

$\forall \vec{y}\left(\varphi(x, \vec{y})\right.$ nonalgebraic $\Longrightarrow \exists x\left(\varphi(x, \vec{y}) \wedge x \in H_{2}\right) \wedge$

$\left.\forall w_{1} \ldots \forall w_{m} \in H_{1} \neg \psi\left(x, w_{1}, \ldots, w_{m}, \vec{y}\right)\right)$

(4) For all $\mathcal{L}_{0}$-formulas $\varphi(x, \vec{y}), m \in \omega$, and all $\mathcal{L}_{0}$-formulas $\psi\left(x, z_{1}, \ldots, z_{m}, \vec{y}\right)$ such that for some $n \in \omega \forall \vec{z} \forall \vec{y} \exists \leq n x \psi(x, \vec{z}, \vec{y}) \quad($ so $\psi(x, \vec{y}, \vec{z})$ is always algebraic in $x)$

$\forall \vec{y}(\varphi(x, \vec{y})$ nonalgebraic $\Longrightarrow \exists x \varphi(x, \vec{y}) \wedge$

$\left.\forall w_{1} \ldots \forall w_{m} \in H_{1} \cup H_{2} \neg \psi\left(x, w_{1}, \ldots, w_{m}, \vec{y}\right)\right)$

Furthermore, if $\left(M, H_{1}, H_{2}\right) \models T^{\text {tri }}$ is $|T|^{+}$-saturated, then $\left(M, H_{1}, H_{2}\right)$ is an $H$-triple.

Thus when $T_{0}$ is a supersimple $S U$-rank one theory, $T_{\mathcal{G}}^{i n d}=T^{t r i}$ is first order.

3.6. $H$ structures of lovely pairs of $S U$-rank one theories. Let $T$ be a supersimple theory of SU-rank 1, $T_{P}$ its lovely pairs expansion, and let

$$
\operatorname{cl}(-)=\operatorname{acl}(-\cup P(M))
$$

be the small closure operator in a lovely pair $(M, P)$. Let $\mathcal{G}$ be the formula $\neg P(x)$ in $T_{P}$ (representing $M \backslash P(M)$ ).

Our goal is to expand $T_{P}$ to a theory $T_{P}^{i n d}$ of $H$-structures of $T_{P}$ with respect to $\mathcal{G}$, in the language $\mathcal{L}_{P H}=\mathcal{L}_{P} \cup\{H\}$. Note that the notion of being large in 
the sense of $\mathcal{G}$ coincides with the corresponding notion induced by the small closure operator cl.

The following definition is analogous to Definition 3.1.

Definition 3.4. We say that an $\mathcal{L}_{P H}$-structure $(M, P, H)$ is a $P H$-structure of $T$ if

(1) $P(M)$ is an elementary substructure of $M$;

(2) $H(M)$ is acl-independent over $P(M)$;

(3) for any non-algebraic type $q \in S_{1}^{T}(A)$ over a finite set $A \subset M, q$ is realized in

(density of $P$ over $H) P(M) \backslash \operatorname{acl}(H(M) A)$;

(density of $H$ over $P) H(M) \backslash \operatorname{acl}(P(M) A$ );

(extension) $M \backslash \operatorname{acl}(P(M) H(M) A)$.

Remark 3.5. (a) It suffices to require $P(M)$ to be dense in the usual sense, i.e. $q$ having a realization in $P(M)$. Indeed, $H(M)$ is acl-independent over $P(M)$, and if $A$ is finite, then for some finite $\vec{h} \subset H(M)$ we have $H(M) \downarrow_{\vec{h} P(M)} A P(M)$. Hence $H(M) \backslash \vec{h}$ is acl-independent over $A P(M)$. Clearly, if $P(M)$ is dense, $q$ can be realized by $b \in P(M) \backslash \operatorname{acl}(\vec{h} A)$. Then $b \notin \operatorname{acl}(A H(M))$.

(b) We can get a PH-structure from an $H$-triple $\left(M, H_{1}, H_{2}\right)$ (see previous example), by letting $P(M)=\operatorname{acl}\left(H_{1}\right)$.

(c) A usual elementary chain argument shows that any $L_{P H}$ structure $(M, P, H)$ satisfying (1,2) embeds in a $P H$-structure $(N, P, H)$ so that $H(N) \downarrow_{H(M)} M P(N)$ and $P(N) \downarrow_{P(M)} M H(N)$. In particular, $P H$-structures exist.

(d) Reducts $(M, P)$ and $(M, H)$ of $(M, P, H)$ are lovely pairs and $H$-structures, respectively.

Proposition 3.6. $(M, P, H)$ is an $H$-structure of $T_{P}$ (with respect to $\mathcal{G}$ ) if and only if $(M, P, H)$ is a $P H$-structure.

Proof. Assume first that $(M, P, H)$ is an $H$-structure. Then the pair $(M, P)$ is lovely and thus $(M, P, H)$ satisfies the density property for $P$. Now let $A \subset M$ be finite and let $q \in S_{1}(A)$ be non-algebraic. Let $\hat{q} \in S_{1}^{P}(A)$ be an extension of $q$ that contains no small formula with parameters in $A$. Then by the density/coheir property for cl it follows that there is $a \in H(M)$ such that $a \models \hat{q}$. In particular, $a \models q$ and $a \notin \operatorname{cl}(A)$ and thus we get the density property for $H$ over $P$. Finally, by extension property, there exists $c \in M$ realizing $\hat{q}$ such that $c \downarrow_{A}^{P} H(M)$ (here independence is in the sense of $\left.T_{P}\right)$. Then $c \notin \operatorname{cl}(A \cup H(M))=\operatorname{acl}(A \cup P(M) \cup H(M))$. Thus the extension property of $P H$-structures holds as well.

Now assume that $(M, P, H)$ is a $P H$-structure. Then by the density property for $P$ and the extension property it follows that $(M, P)$ is a lovely pair, and $H(M)$ is a cl-independent set (hence, forking independent in the sense of $T_{P}$ ). Now let $A \subset M$ be finite and let $\hat{q} \in S_{1}^{P}(A)$ be large. We may enlarge $A$ and assume that $A$ is $P$-independent. Let $q$ be the restriction of $\hat{q}$ to the language $\mathcal{L}$. Note that $\hat{q}$ is the unique extension of $q$ to a non-small type. By the density for $H$ over $P$, there is $a \in H(M)$ such that $a \models q, a \notin \operatorname{cl}(A)$ and thus $a \models \hat{q}$. This proves the density/coheir property of $H$-structures.

To prove the extension property of $H$-structures, we start with $a \in M$ and a finite $A \subset M$. We may assume that $A$ is $P$-independent.If $a$ is large over $A$ 
in $T_{P}$, then by the extension property for $P H$-structures, there is $a^{\prime} \in M$ such that $\operatorname{tp}\left(a^{\prime} / A\right)=\operatorname{tp}(a / A)$ and $a^{\prime} \notin \operatorname{acl}(P(M) H(M))$. Then $\operatorname{tp}^{P}\left(a^{\prime} / A\right)=\operatorname{tp}^{P}(a / A)$ (where tp ${ }^{P}$ denotes the type in the sense of $T_{P}$ ) and $a^{\prime} \downarrow_{A}^{P} H(M)$, as needed.

Now, suppose $a$ is small over $A$ in $T_{P}$. Thus, $a \in \operatorname{acl}(A P(M))$. Then it suffices to show that for any tuple $\vec{b} \in P(M)$, acl-independent over $A$, there exists $\vec{b}^{\prime} \in P(M)$ such that $\operatorname{tp}\left(\vec{b}^{\prime} / A\right)=\operatorname{tp}(\vec{b} / A)$ and $\vec{b}^{\prime} \downarrow_{A}^{P} H(M)$. We may also assume that $\vec{b}=b$ is a single element. Suppose for any $b^{\prime} \in P(M)$ realizing $\operatorname{tp}(b / A)$ we have that $\operatorname{tp}^{P}\left(\vec{b}^{\prime} / A H(M)\right)$ forks over $A$. Since $P(M)$ has SU-rank 1 , this means that $b^{\prime} \in$ $\operatorname{acl}^{P}(A H(M))$ for any $b^{\prime} \in P(M)$ realizing tp $(b / A)$. Next, as in Remark 3.5(a), we have $H(M) \downarrow_{\vec{h} P(M)} A P(M)$. Since $H(M)$ is acl-independent over $P(M)$, we also have $H(M) \downarrow_{\vec{h}} A P(M)$. Let $\vec{c} \in P(M)$ be a finite tuple such that $A \vec{h} \downarrow \vec{c} P(M)$. Then $A H(M) \downarrow_{\vec{c}} P(M)$. Hence, $A H(M) \vec{c}$ is $P$-independent, and $\operatorname{acl}^{P}(A H(M) \vec{c})=$ $\operatorname{acl}(A H(M) \vec{c})$. Therefore, $b^{\prime} \in \operatorname{acl}(A H(M) \vec{c})$ for any $b^{\prime} \in P(M)$ realizing $\operatorname{tp}(b / A)$. But by density of $P$ over $H$, we can find $b^{\prime} \in P(M)$ realizing a nonalgebraic extension of $\operatorname{tp}(b / A)$ to $A \vec{c}$ such that $b^{\prime} \notin \operatorname{acl}(A \vec{c} H(M))$, a contradiction.

We will now show that the class of $P H$-structures is "first order", that is, that there is a set of axioms whose $|T|^{+}$-saturated models are the $P H$-structures. The axiomatization works as in $H$-triples.

Proposition 3.7. Assume $T$ eliminates $\exists^{\infty}$. Then the theory $T_{P H}$ is axiomatized by:

(1) $T$

(2) axioms saying that $P$ distinguishes an elementary substructure.

(3) For all $\mathcal{L}$-formulas $\varphi(x, \vec{y})$

$\forall \vec{y}(\varphi(x, \vec{y})$ nonalgebraic $\Longrightarrow \exists x(\varphi(x, \vec{y}) \wedge x \in P))$.

(4) For all $\mathcal{L}$-formulas $\varphi(x, \vec{y}), m \in \omega$, and all $\mathcal{L}$-formulas $\psi\left(x, z_{1}, \ldots, z_{m}, \vec{y}\right)$ such that for some $n \in \omega \forall \vec{z} \forall \vec{y} \exists \leq n x \psi(x, \vec{z}, \vec{y})$ (so $\psi(x, \vec{y}, \vec{z})$ is always algebraic in $x)$

$\forall \vec{y}(\varphi(x, \vec{y})$ nonalgebraic $\Longrightarrow \exists x(\varphi(x, \vec{y}) \wedge x \in H) \wedge$

$\left.\forall w_{1} \ldots \forall w_{m} \in P \neg \psi\left(x, w_{1}, \ldots, w_{m}, \vec{y}\right)\right)$

(5) For all $\mathcal{L}$-formulas $\varphi(x, \vec{y}), m \in \omega$, and all $\mathcal{L}$-formulas $\psi\left(x, z_{1}, \ldots, z_{m}, \vec{y}\right)$ such that for some $n \in \omega \forall \vec{z} \forall \vec{y} \exists \leq n x \psi(x, \vec{z}, \vec{y})$ (so $\psi(x, \vec{y}, \vec{z})$ is always algebraic in $x)$

$\forall \vec{y}(\varphi(x, \vec{y})$ nonalgebraic $\Longrightarrow \exists x(\varphi(x, \vec{y}) \wedge x \notin P \wedge x \notin H) \wedge$

$\left.\forall w_{1} \ldots \forall w_{m} \in P \cup H \neg \psi\left(x, w_{1}, \ldots, w_{m}, \vec{y}\right)\right)$

Furthermore, if $(M, P, H) \models T_{P H}$ is $|T|^{+}$-saturated, then $(M, P, H)$ is a PH-structure.

Now we list a family of structures of $S U$-rank $\omega$ where we do not know if the corresponding theory of $H$-structures is axiomatizable. In all three cases it is open whether or not the extension property is first order.

3.7. Vector space with a generic automorphism. Let $T$ be the theory of an infinite vector space over a division ring $F$; it is strongly minimal, has DMP and quantifier elimination. Recall that the definable closure of a set inside models of $T$ corresponds to the linear span of the set. For a tuple $\vec{a}$ in a model of $T$ we write $\operatorname{qftp}^{-}(\vec{a})$ for its quantifier free type (which isolates the type) and $\mathrm{dcl}^{-}(\vec{a})$ 
for its definable closure. Let $\sigma$ be a new function symbol and let $T_{\sigma}$ be theory $T$ together with axioms stating that $\sigma$ is a generic automorphism. The notes 18 deal with strongly minimal theories with a generic automorphism, the theory $T_{\sigma}$ appears there as an example. In particular it is shown in [18, Proposition 3.9] how to axiomatize $T_{\sigma}$. By [18, Corollary 4.14 and Lemma 4.16] the theory has $S U$-rank $\omega$ and by [18, Example 5.7] it is stable. It is also proved in [18, Remark 3.15] that for $(M, \sigma) \models T_{\sigma}$ and $\vec{a} \in M$, the algebraic closure of $\vec{a}$ in $T_{\sigma}$ denoted by $\operatorname{dcl}(\vec{a})$ corresponds to $\mathrm{dcl}^{-}\left(\left\{\sigma^{i}(\vec{a}): i \in \mathbb{Z}\right\}\right)$

We claim that $T_{\sigma}$ has quantifier elimination. Indeed, let $(M, \sigma) \models T_{\sigma}$ be saturated and let $\vec{a}, \vec{a}^{\prime} \in(M, \sigma)$ be such that $q f t p^{-}\left(\ldots, \sigma^{-1}(\vec{a}), \vec{a}, \sigma(\vec{a}), \sigma^{2}(\vec{a}), \ldots\right)=$ $q f p^{-}\left(\ldots, \sigma^{-1}\left(\vec{a}^{\prime}\right), \vec{a}^{\prime}, \sigma\left(\vec{a}^{\prime}\right), \sigma^{2}\left(\vec{a}^{\prime}\right), \ldots\right)$. Let $c \in M$, we will find $c^{\prime} \in M$ such that $q f t p^{-}\left(\ldots, \sigma^{-1}(\vec{a} c), \vec{a} c, \sigma(\vec{a} c), \sigma^{2}(\vec{a} c), \ldots\right)=q f t p^{-}\left(\ldots, \sigma^{-1}\left(\vec{a}^{\prime} c^{\prime}\right), \vec{a}^{\prime} c^{\prime}, \sigma\left(\vec{a}^{\prime} c^{\prime}\right), \sigma^{2}\left(\vec{a}^{\prime} c^{\prime}\right), \ldots\right)$. Consider the substructure $\operatorname{dcl}(\vec{a}, c)$ and the free amalgamation of $(M, \sigma)$ with $\operatorname{dcl}(\vec{a}, c)$ that identifies the substructure $\operatorname{dcl}\left(\vec{a}^{\prime}\right)$ of $M$ with the substructure $\operatorname{dcl}(\vec{a})$ of $\operatorname{dcl}(\vec{a} c)$. Then this amalgamation is a superstructure of $(M, \sigma)$ and the copy of $c$ in the amalgamation has the desired type. Now use that $(M, \sigma)$ is existentially closed and saturated to get $c^{\prime}$.

The theory $T_{\sigma}$ has a unique type of $U$-rank $\omega$, namely the type of a transformally independent element. Take $\mathcal{G}$ to be this type, which is definable over $\emptyset$. By definability of types, $T_{\sigma}$ eliminates the quantifier $\exists^{\text {large }}$.

Question Is the extension property first order for $T_{\sigma}$ ?

We can choose other partial types for $\mathcal{G}$. For example, let $\mathcal{G}$ be $\sigma(x)=x$, $x \notin \mathrm{dcl}^{-}(\emptyset)$. By quantifier elimination and stability the set $\sigma(x)=x$ is strongly minimal. The partial type $\mathcal{G}$ corresponds to the generic type of this strongly minimal set, which is stationary and definable over $\emptyset$. The definability of the type implies elimination of $\exists^{\text {large }}$.

3.8. ACFA. Let $T=A C F A$, (a completion) of the theory of algebraically closed fields with a generic automorphism. This theory is simple of $S U$-rank $\omega$ and it is unstable.

Let $p(x)$ be the generic type of the theory, namely the type of a transformally independent element. This type is complete, stationary and definable over $\emptyset$. Let $\mathcal{G}=p(x)$. Let $\varphi(x, \vec{y})$ be a formula and let $\psi(\vec{y})$ be its $p$-definition. Then for $(K, \sigma) \models A C F A, \vec{a} \in K$, the formula $\varphi(x, \vec{a})$ is large iff $\psi(\vec{a})$. Thus this theory eliminates the quantifier $\exists^{\text {large }}$.

Question Is the extension property first order for ACFA? Does ACFA satisfy wnfcp?

3.9. Hrushovski amalgamation without collapsing. In this subsection we follow the presentation of Hrushovski amalgamations from [21, all the results we mention can be found in 21. Let $\mathcal{L}=\{R\}$ where $R$ stands for a ternary relation. We let $\mathcal{C}$ be the class of $\mathcal{L}$-structures where $R$ is symmetric and not reflexive. For $A \in \mathcal{C}$ a finite structure we let $\delta(A)=|A|-|R(A)|$ and we let $\mathcal{C}_{\text {fin }}^{0}$ be the subclass of $\mathcal{C}$ consisting of all finite $\mathcal{L}$-structures $M$ where for $A \subset M$ we have $\delta(A) \geq 0$. Finally $M^{0}$ stands for the Fraïssé limit of the class $\mathcal{C}_{\text {fin }}^{0}$. Let $T_{0}$ be the theory of $M^{0}$, then $M R\left(T_{0}\right)=\omega$ and $M d\left(T_{0}\right)=1$.

Now let $M \models T_{0}$ and for $A \subset M$ finite we define $d(A)=\inf \{\delta(B): A \subset B\}$. Then $d$ is the dimension function of a pregeometry and that for an element $a$ and a set $B, d(a / B)=1$ if and only if $M R(a / B)=\omega$ if and only if $U(a / B)=\omega$. The 
type $p(x)$ described by $d(x)=1$ is stationary and definable over $\emptyset$, if we choose $\mathcal{G}=p(x)$ then $T_{0}$ eliminates the quantifier $\exists^{\text {large }}$.

Question Is the extension property first order for $T_{0}$ ? Does $T_{0}$ satisfy nfcp?

\section{Definable sets in $H$-Structures}

Fix $T$ a supersimple theory, $\mathcal{G}$ a type-definable set over $\emptyset$, and assume that $T$ eliminates $\exists^{\text {large }}$ and that the extension property is first order. Let $(M, H(M)) \models$ $T_{\mathcal{G}}^{i n d}$. Our next goal is to obtain a description of definable subsets of $M$ and $H(M)$ in the language $\mathcal{L}_{H}$.

Notation 4.1. Let $(M, H(M))$ be an $H$-structure. Let $\vec{a}$ be a tuple in $M$. We denote by $\operatorname{etp}_{H}(\vec{a})$ the collection of formulas of the form $\exists x_{1} \in H \ldots \exists x_{m} \in H \varphi(\vec{x}, \vec{y})$, where $\varphi(\vec{x}, \vec{y})$ is an $\mathcal{L}$-formula such that there exists $\vec{h} \in H$ with $M \models \varphi(\vec{h}, \vec{a})$.

The next results appears in [6] in the setting of geometric theories. The proof we present here is a translation of Corollary 3.11 [2] to the setting of $H$-structures.

Lemma 4.2. Let $(M, H(M)),(N, H(N))$ be $H$-structures. Let $\vec{a}, \vec{b}$ be tuples of the same arity from $M, N$ respectively. Then the following are equivalent:

(1) $\operatorname{etp}_{H}(\vec{a})=\operatorname{etp}_{H}(\vec{b})$.

(2) $\vec{a}, \vec{b}$ have the same $\mathcal{L}_{H}$-type.

Proof. Clearly (2) implies (1). Assume (1), then $\operatorname{tp}(\vec{a})=\operatorname{tp}(\vec{b})$.

Let $\vec{h}_{\vec{a}}$ be an enumeration of a finite subset of $H(M)$ such that $\operatorname{tp}(\vec{a} / H(M))$ does not fork over $\vec{h}_{\vec{a}}$. Let $q(\vec{z}, \vec{a})=\operatorname{tp}\left(\vec{h}_{\vec{a}}, \vec{a}\right)$. Since $\operatorname{etp}_{H}(\vec{a})=\operatorname{etp}_{H}(\vec{b}), q(z, \vec{b})$ is finitely satisfiable in $H(N)$, and since $(N, H(N))$ is an $H$-structure, it is realized in $H(N)$ say by $\vec{h}_{\vec{b}}$.

Claim $\operatorname{tp}(\vec{b} / H(N))$ does not fork over $\vec{h}_{\vec{b}}$.

Suppose, in order to get a contradiction, that $t p(\vec{b} / H(N))$ forks over $\vec{h}_{\vec{b}}$. Then there are $\mathcal{L}$-formulas $\varphi(\vec{x}, \vec{y}), \psi(\vec{x}, \vec{z}), k<\omega$, and $\vec{c} \in H(N)$ such that $\psi(\vec{b}, \vec{c})$ holds and $D(\psi(\vec{x}, \vec{c}), \varphi, k)<n$ where $n=D\left(\operatorname{tp}\left(\vec{b} / \vec{h}_{\vec{b}}\right), \varphi, k\right)$. Now there is a formula $\chi(\vec{z}) \in \operatorname{tp}(\vec{c})$ such that for any $\vec{c}^{\prime} \models \chi(\vec{z}), D\left(\psi\left(\vec{x}, \vec{c}^{\prime}\right), \varphi, k\right)<n$. As $\operatorname{etp}_{H}(\vec{a})=\operatorname{etp}_{H}(\vec{b})$, there is $\vec{c}^{\prime} \in H(M)$ such that $\psi\left(\vec{a}, \vec{c}^{\prime}\right) \wedge \chi\left(\vec{c}^{\prime}\right)$, implying that $\operatorname{tp}(\vec{a} / H(M))$ forks over $\vec{h}_{\vec{a}}$, a contradiction. So $t p(\vec{b} / H(N))$ does not fork over $\vec{h}_{\vec{b}}$. Note that $\operatorname{tp}\left(\vec{a} \vec{h}_{\vec{a}}\right)=\operatorname{tp}\left(\vec{b} \vec{h}_{\vec{b}}\right)$ and both tuples are $H$-independent, so by Proposition $2.7 \operatorname{tp}_{H}(\vec{a})=\operatorname{tp}_{H}(\vec{b})$.

Note that Lemma 4.2 hold for tuples in $M^{e q}$.

The previous result implies near model completeness: every $\mathcal{L}_{H}$-definable set can be written as a boolean combination of formulas of the form $\exists x_{1} \in H \ldots \exists x_{m} \in$ $H \varphi(\vec{x}, \vec{y})$, where $\varphi(\vec{x}, \vec{y})$ is an $\mathcal{L}$-formula. We will refine this result, starting by understanding the $\mathcal{L}_{H}$-definable subsets of $H(M)$. This material is very similar to the results presented in [6].

Lemma 4.3. Let $\left(M_{0}, H\left(M_{0}\right)\right) \preceq\left(M_{1}, H\left(M_{1}\right)\right)$ and assume that $\left(M_{1}, H\left(M_{1}\right)\right)$ is $\left|M_{0}\right|$-saturated. Then $M_{0}$ (seen as a subset of $M_{1}$ ) is an $H$-independent set.

Proof. Assume not. Then there is $\vec{a} \in M_{0}$ such that $\vec{a} \bigotimes_{H\left(M_{0}\right)} H\left(M_{1}\right)$. Let

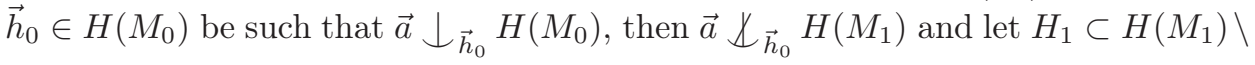


$H\left(M_{0}\right)$ be finite and $h \in H\left(M_{1}\right) \backslash\left(H\left(M_{0}\right) \cup H_{1}\right)$ such that $\vec{a} \mathbb{X}_{\vec{h}_{0}} H_{1} h$, but $\vec{a} \downarrow_{\vec{h}_{0}} H_{1}$. So $h$ forks with $\vec{a} \vec{h}_{0} H_{1}$. Let $\varphi\left(x, \vec{a} ; \vec{h}_{0}, H_{1}\right)$ be a formula which holds for $h$ such that $\varphi\left(x, \vec{a} ; \vec{h}_{0}, H_{1}\right) \wedge \mathcal{G}(x)$ forks over $\emptyset$. Since $\left(M_{0}, H\left(M_{0}\right)\right) \preceq\left(M_{1}, H\left(M_{1}\right)\right)$ there is $h_{2} \in H\left(M_{0}\right)$ and $H_{2} \subset H\left(M_{0}\right)$ both disjoint from each other and disjoint from $\vec{h}_{0}$ such that $\varphi\left(h_{2}, \vec{a}, \vec{h}_{0}, H_{2}\right) \wedge \neg \exists^{\text {large }} x \varphi\left(x, \vec{a}, \vec{h}_{0}, H_{2}\right)$ holds, so $\vec{a} \mathbb{Z}_{\vec{h}_{0}} h_{2} H_{2}$, a contradiction.

Proposition 4.4. Let $(M, H(M))$ be an $H$-structure and let $Y \subset H(M)^{n}$ be $\mathcal{L}_{H^{-}}$ definable. Then there is $X \subset M^{n} \mathcal{L}$-definable such that $Y=X \cap H(M)^{n}$.

Proof. Let $\left(M_{1}, H\left(M_{1}\right)\right) \succeq(M, H(M))$ be $\kappa$-saturated where $\kappa>|M|+|L|$ and let $\vec{a}, \vec{b} \in H\left(M_{1}\right)^{n}$ be such that $\operatorname{tp}(\vec{a} / M)=\operatorname{tp}(\vec{b} / M)$. We will prove that $\operatorname{tp}_{H}(\vec{a} / M)=$ $\operatorname{tp}_{H}(\vec{b} / M)$ and the result will follow by compactness. Since $\vec{a}, \vec{b} \in H\left(M_{1}\right)^{n}$, we get by Lemma 4.3 that $M \vec{a}, M \vec{b}$ are $H$-independent sets and thus by Lemma 2.7 we get $\operatorname{tp}_{H}(\vec{a} / M)=\operatorname{tp}_{H}(\vec{b} / M)$.

Proposition 4.5. Let $(M, H(M))$ be an $H$-structure. Let $\vec{a} \in M$. Then there is a unique smallest subset $H_{0} \subset H(M)$ such that $\vec{a} \downarrow_{H_{0}} H$.

Proof. Since $T$ is supersimple, there is a finite subset $H_{1} \subset H(M)$ such that $\vec{a} \downarrow_{H_{1}} H(M)$. Choose such subset so that $\left|H_{1}\right|$ (the size of the subset) is minimal. We will now show such a set $H_{1}$ is unique.

If $\vec{a} \downarrow H(M)$, then $H_{1}=\emptyset$ and the result follows. So we may assume that $\vec{a} \not H(M)$.

Let $H_{2} \subset H(M)$ be another minimal finite subset such that $\vec{a} \downarrow_{H_{2}} H(M)$ and let $H_{0}=H_{1} \cap H_{2}$.

Claim $\vec{a} \downarrow_{H_{0}} H(M)$.

Since the elements in $H(M)$ are independent over $\emptyset$, we have $H_{1} \downarrow_{H_{0}} H_{2}$. Since $\vec{a} \downarrow_{H_{1}} H(M)$ we also have $\vec{a} \downarrow_{H_{1}} H_{2}$ and by transitivity $\vec{a} \downarrow_{H_{0}} H_{2}$. But $\vec{a} \downarrow_{H_{2}} H(M)$, so using again transitivity we get $\vec{a} \downarrow_{H_{0}} H(M)$.

Finally by minimality of $\left|H_{1}\right|$ we get that $H_{0}=H_{1}=H_{2}$ as we wanted.

Remark 4.6. Let $(M, H(M))$ be an $H$-structure. Let $\vec{a} \in M$ and let $C \subset M$ be $H$-independent. Then whenever $H_{1}, H_{2}$ are finite subsets of $H$ and $H_{0}=H_{1} \cap H_{2}$, we have $H_{1} \downarrow_{H_{0} C} H_{2}$. Then the argument from the previous proposition relativized to $C$ shows that there is a unique smallest subset $H_{0} \subset H(M)$ such that $\vec{a} \downarrow_{H_{0} C} H$.

Notation 4.7. Let $(M, H(M))$ be an $H$-structure. Let $\vec{a} \in M$. Let $H_{0} \subset H(M)$ be the smallest subset such that $\vec{a} \downarrow_{H_{0}} H$. We call $H_{0}$ the $H$-basis of $\vec{a}$ and we denote it as $H B(\vec{a})$. Given $C \subset M$ such that $C$ is $H$-independent, let $H_{1} \subset H(M)$ be the smallest subset such that $\vec{a} \downarrow_{C_{H_{1}}} H$. We call $H_{1}$ the $H$-basis of $\vec{a}$ over $C$ and we denote it as $H B(\vec{a} / C)$. Note that $H$-basis is a finite set, if we give it an order to view it as a tuple, we will explicitly say so. Finally note that we can also define the $H$-basis for $\vec{a} \in M^{e q}$.

Proposition 4.8. Let $(M, H(M))$ be an $H$-structure. Let $a_{1}, \ldots, a_{n}, a_{n+1} \in M$ and let $C \subset M$ be such that $C$ is $H$-independent. Then $H B\left(a_{1}, \ldots, a_{n}, a_{n+1} / C\right)=$ $H B\left(a_{1}, \ldots, a_{n} / C\right) \cup H B\left(a_{n+1} / C a_{1}, \ldots, a_{n} H B\left(a_{1}, \ldots, a_{n} / C\right)\right)$. 
Proof. Let $H_{1}=H B\left(a_{1}, \ldots, a_{n} / C\right)$. First note that since $a_{1}, \ldots, a_{n} \downarrow_{C H_{1}} H$, then the set $a_{1}, \ldots, a_{n} C H_{1}$ is $H$-independent and we can define $H_{2}=H B\left(a_{n+1} / C a_{1}, \ldots, a_{n} H_{1}\right)$.

Finally, let $H_{0}=H B\left(a_{1}, \ldots, a_{n}, a_{n+1} / C\right)$.

Claim $H_{0} \subset H_{1} H_{2}$.

We have $a_{1}, \ldots, a_{n} \downarrow_{C H_{1}} H$ and $a_{n+1} \downarrow_{C H_{1} H_{2} a_{1} \ldots, \ldots a_{n}} H$, so by transitivity,

$a_{1}, \ldots, a_{n} a_{n+1} \downarrow_{\mathrm{CH}_{1} \mathrm{H}_{2}} H$ and by the minimality of an $H$-basis, we have $H_{0} \subset$ $\mathrm{H}_{1} \mathrm{H}_{2}$.

Claim $H_{0} \supset H_{1} H_{2}$.

By definition, $a_{1}, \ldots, a_{n} a_{n+1} \downarrow_{C H_{0}} H$, so $a_{1}, \ldots, a_{n} \downarrow_{C H_{0}} H$ and by minimality we have $H_{1} \subset H_{0}$. We also get by transitivity that $a_{n+1} \downarrow_{C a_{1}, \ldots, a_{n} H_{1} H_{0}} H$ and by the minimality of $H$-basis we get $H_{2} \subset H_{0}$ as desired.

Proposition 4.9. Let $(M, H(M))$ be an $H$-structure. Let $\vec{a} \in M$ and let $C \subset D \subset$ $M$ be such that $C, D$ are $H$-independent. Then $H B(\vec{a} / C) \subset H B(D) \cup H B(\vec{a} / D)$.

Proof. Write $H_{\vec{a}}=H B(\vec{a} / D)$. Then $\vec{a} D \downarrow_{H_{\vec{a}} H(D)} H$ and $\vec{a} \downarrow_{H_{\vec{a}} C H(D)} H$. By minimality of $H B(\vec{a} / C)$ we get that $H B(\vec{a} / C) \subset H_{\vec{a}} \cup H(D)$ and thus if $h \in$ $H B(\vec{a} / C) \backslash H_{\vec{a}}$, we must have $h \in H(D)$.

The following definition and proposition were very fruitfull to show the preservation of NTP2 to $T^{\text {ind }}$ when $T$ was geometric see 3 .

Definition 4.10. Let $(M, H) \models T_{\mathcal{G}}^{\text {ind }}$ be saturated. We say that an $\mathcal{L}_{H}$-formula $\psi(x, \vec{c})$ defines a $H$-large subset of $M$ if there is $b \models \psi(x, \vec{c})$ such that $b \downarrow H(M) \vec{c}$, $b \models \mathcal{G}$. Otherwise we say that $\psi(x, \vec{c})$ defines a $H$-small subset of $M$. Note that the formula $\psi(x, \vec{c})$ is $H$-large if there are infinitely many realizations of $\psi(x, \vec{c})$ in $\mathcal{G}$ that are independent from $H(M) \vec{c}$. For $A \subset M$ and $b \in M$, we say that $b$ is $H$-small over $A$ if it satisfies an $H$-small formula $\psi(x, \vec{a})$ with $\vec{a} \in A$, otherwise we say that $b$ is $H$-large over $A$.

Proposition 4.11. Let $(M, H(M))$ be an $H$-structure and let $Y \subset M$ be $\mathcal{L}_{H}$ definable. Then there is $X \subset M \mathcal{L}$-definable such that $Y \triangle X$ is $H$-small, where $\triangle$ stands for a boolean connective for the symmetric difference.

Proof. If $Y$ is $H$-small or its complement is $H$-small, the result is clear, so we may assume that both $Y$ and $M \backslash Y$ are $H$-large. Assume that $Y$ is definable over $\vec{a}$ and that $\vec{a}=\vec{a} H B(\vec{a})$. Let $b \in Y$ be such that $b \models \mathcal{G}, b \downarrow H(M) \vec{a}$ and let $c \in M \backslash Y$ be such that $c \models \mathcal{G}, c \downarrow H(M) \vec{a}$. Then $b \vec{a}, c \vec{a}$ are $H$-independent and thus there is $X_{b c}$ an $\mathcal{L}$-definable set such that $b \in X_{b c}$ and $c \notin X_{b c}$. By compactness, we may get a single $\mathcal{L}$-definable set $X$ such that for all $b^{\prime} \in Y$ and $c^{\prime} \in M \backslash Y H$-large over $\vec{a}$, we have $b^{\prime} \in X$ and $c^{\prime} \in M \backslash X$. This shows that $Y \triangle X$ is $H$-small.

Our next goal is to characterize the algebraic closure in $H$-structures. The key tool is the following result:

Lemma 4.12. Let $(M, H(M))$ be an $H$-structure, and let $A \subset M$ be acl-closed and $H$-independent. Then $A$ is acl $_{H}$-closed.

Proof. Suppose $a \in M, a \notin A$. We do the argument by cases:

Case 1. Assume $a \downarrow_{A} H(M)$. Using repeatedly the extension property, we can find $\left\{a_{i}: i \in \omega\right\}$ all of them realizing $\operatorname{tp}(a / A)$ and independent over $A \cup H(M)$. By Proposition 2.7. each $a_{i}$ realizes $\operatorname{tp}_{H}(a / A)$, and thus $a \notin \operatorname{acl}_{H}(A)$. 
Case 2. Assume $a \swarrow_{A} H(M)$. Let $\vec{h} \in H(M)$ be an enumeration of $H B(a / A)$ (so we see it as a tuple), then $a \downarrow_{A \vec{h}} H(M)$. Using the density property we can find $\left\{\vec{h}_{i}: i \in \omega\right\}$ in $H$ all of them realizing $\operatorname{tp}(\vec{h} / A)$ and disjoint one from the other. Let $a_{i} \in M$ be such that $\operatorname{tp}_{H}\left(a_{i}, \vec{h}_{i} / A\right)=\operatorname{tp}_{H}(a, \vec{h} / A)$. Then for each $i, H B\left(a_{i} / A\right)=\vec{h}_{i}$ (again with an order) and so the family $\left\{a_{i}: i \in \omega\right\}$ is infinite and they all satisfy $t p_{H}(a / A)$.

Corollary 4.13. Let $(M, H(M))$ be an $H$-structure, and let $A \subset M$. Then $\operatorname{acl}_{H}(A)=\operatorname{acl}(A, H B(A))$.

Proof. By Proposition 4.5, it is clear that $H B(A) \subset \operatorname{acl}_{H}(A)$, so $\operatorname{acl}_{H}(A) \supset$ $\operatorname{acl}(A, H B(A))$. On the other hand, $A \cup H B(A)$ is $H$-closed, so by the previous proposition, $\operatorname{acl}(A \cup H B(A))=\operatorname{acl}_{H}(A \cup H B(A))$ and thus $\operatorname{acl}_{H}(A) \subset$ $\operatorname{acl}(A, H B(A))$

\section{SuPERSIMPLICITY}

Fix $T$ a supersimple theory, $\mathcal{G}$ a type definable set over $\emptyset$, and assume that $T$ eliminates $\exists^{\text {large }}$ and that the extension property is first order. In this section we prove that $T_{\mathcal{G}}^{\text {ind }}$ is supersimple and characterize forking in $T_{\mathcal{G}}^{\text {ind }}$.

Theorem 5.1. The theory $T_{\mathcal{G}}^{\text {ind }}$ is supersimple.

Proof. We will prove that non-dividing has local character.

Let $(M, H(M)) \models T_{\mathcal{G}}^{\text {ind }}$ be saturated. Let $C \subset D \subset M$ and assume that $C=\operatorname{acl}_{H}(C)$ and $D=\operatorname{acl}_{H}(D)$. Note that both $C$ and $D$ are $H$-independent. Let $\vec{a} \in M$. We will find a collection of conditions for the type of $\vec{a}$ over $C$ that guarantee that $\operatorname{tp}_{H}(\vec{a} / D)$ does not divide over $C$.

Assume that the following conditions hold for $C$ :

(1) $H B(\vec{a} / D)=H B(\vec{a} / C)$.

(2) $\vec{a} \downarrow_{C H(M)} D H(M)$

Claim $\operatorname{tp}_{H}(\vec{a} / D)$ does not divide over $C$.

Let $\left\{D_{i}: i \in \omega\right\}$ be an $\mathcal{L}_{H}$-indiscernible sequence over $C$. Let $\vec{h}$ be an enumeration of $H B(\vec{a} / D)$ and let $q(\vec{x}, D)=\operatorname{tp}(\vec{h}, D)$. Since $D$ is $H$-independent, the tuple $\vec{h}$ is independent over $D$ and all components of $\vec{h}$ realize $\mathcal{G}$. Thus we can find $\vec{g} \models \cup_{i \in \omega} q\left(\vec{x}, D_{i}\right)$ such that $\left\{\vec{g} D_{i}: i \in \omega\right\}$ is indiscernible and $\vec{g}$ is an independent tuple over $\cup_{i \in \omega} D_{i}$. All components of $\vec{g}$ realize $\mathcal{G}$. By the generalized density property, we may assume that $\vec{g}$ is in $H$. Note that $\vec{h} D$ is $H$-independent and $\vec{g} D_{i}$ is also $H$-independent for any $i \in \omega$. So by Proposition $2.7 \operatorname{tp}_{H}(\vec{h} D)=\operatorname{tp}_{H}\left(\vec{g} D_{i}\right)$ for any $i \in \omega$.

Let $\vec{e}$ be such that $\operatorname{tp}_{H}(\vec{a}, D, \vec{h})=\operatorname{tp}_{H}\left(\vec{e}, D_{0}, \vec{g}\right)$. Let $p(\vec{x}, D, \vec{z})=\operatorname{tp}(\vec{a}, D, \vec{h})$. Then $\vec{e} \models p\left(\vec{x}, D_{0}, \vec{g}\right)$. Since $\vec{a} \downarrow_{C H(M)} D H(M)$, we get that $\vec{a} \downarrow_{C \vec{h}} D \vec{h}$ and so $\vec{e} \downarrow_{C \vec{g}} D_{0} \vec{g}$. Since $\left\{\vec{g} D_{i}: i \in \omega\right\}$ is an $\mathcal{L}$-indiscernible sequence over $C \vec{g}$, we can find $\vec{a}^{\prime} \models \cup_{i \in \omega} p\left(\vec{x}, D_{i}, \vec{g}\right)$ such that $\left\{\vec{a}^{\prime} \vec{g} D_{i}: i \in \omega\right\}$ is $\mathcal{L}$-indiscernible and $\vec{a}^{\prime}$ is independent from $\cup_{i \in \omega} D_{i} \vec{g}$ over $C \vec{g}$. By the generalized extension property, we may assume that $\vec{a}^{\prime} \downarrow_{\cup_{i \in \omega} D_{i} \vec{g}} H(M)$ and by transitivity we get $\vec{a}^{\prime} \downarrow_{C \vec{g}} D_{i} H(M)$ and so $\vec{a}^{\prime} \downarrow_{D_{i} \vec{g}} H(M)$ for each $i \in \omega$. Note that $\vec{a} \vec{h} D$ is $H$-independent and $\vec{a}^{\prime} \vec{g} D_{i}$ is also $H$-independent for any $i \in \omega$. Thus, by Proposition 2.7 $\operatorname{tp}_{H}(\vec{a} \vec{h} D)=\operatorname{tp}_{H}\left(\vec{a}^{\prime} \vec{g} D_{i}\right)$ for any $i \in \omega$. 
This shows that $\operatorname{tp}_{H}(\vec{a} / D)$ does not divide over $C$.

Since $T$ is supersimple, for any $D$ and $\vec{a}$ we can always choose a finite subset $C_{0}$ of $D$ such that $C=\operatorname{acl}_{H}\left(C_{0}\right)$ satisfies the conditions (1) and (2) above. This shows that $T_{\mathcal{G}}^{\text {ind }}$ is supersimple.

Notation 5.2. Whenever $(M, H(M)) \models T_{\mathcal{G}}^{\text {ind }}$ is sufficiently saturated and $A, B, C \subset$ $M$, we write $A \downarrow_{C}^{\text {ind }} B$ for $\operatorname{tp}_{H}(A / B C)$ does not fork over $C$.

Theorem 5.3. Let $(M, H) \models T_{\mathcal{G}}^{\text {ind }}$ be saturated, let $C \subset D \subset M$ be such that $C=\operatorname{acl}_{H}(C), D=\operatorname{acl}_{H}(D)$ and let $\vec{a} \in M$. Then $\operatorname{tp}_{H}(\vec{a} / D)$ does not fork over $C$ if and only if:

(1) $H B(\vec{a} / D)=H B(\vec{a} / C)$.

(2) $\vec{a} \downarrow_{C H(M)} D H(M)$

Proof. In the proof of Theorem 5.1 we showed that if the two conditions listed hold, then $\operatorname{tp}_{H}(\vec{a} / D)$ does not fork over $C$. It remains to show the other direction.

Case 1: Assume that $\vec{a} \ell_{C H(M)} D H(M)$.

Claim $\vec{a} \chi_{C H(D)}^{\text {ind }} D$.

Let $p(\vec{x}, \vec{y}, C H(D))=\operatorname{tp}_{H}(\vec{a}, D / C H(D))$, where we view $D$ as an ordered tuple. Let $\left\{D_{i}: i \in \omega\right\}$ be an $\mathcal{L}$-Morley sequence in $\operatorname{tp}(D / C H(D))$. By the generalized extension property, we may assume that $\left\{D_{i}: i \in \omega\right\}$ is independent from $H(M)$ over $C H(D)$. In particular, for every $i, D_{i} \downarrow_{C H(D)} H(M)$. Since $C \downarrow_{H(C)} H(M)$ (as $C=\operatorname{acl}_{H}(C)$ ) then $C \downarrow_{H(D)} H(M)$ and by transitivity $D_{i} \downarrow_{H(D)} H(M)$, so $H\left(D_{i}\right) \subset H(D)$. Moreover, since $D_{i} \models \operatorname{tp}(D / C H(D))$ and $H(D) \subset D$, we have that $H(D) \subset D_{i}$. Thus $H(D) \subset H\left(D_{i}\right)$, i.e. $H(D)=H\left(D_{i}\right)$.

By Proposition 2.7. $\operatorname{tp}_{H}(D)=\operatorname{tp}_{H}\left(D_{i}\right)$. Furthermore since $\left\{D_{i}: i \in \omega\right\}$ is Morley over $C H(D)$ and independent from $H(M)$ over $C H(D),\left\{D_{i}: i \in \omega\right\}$ is an $\mathcal{L}_{H}$-indiscernible sequence over $C H(D)$. We will show that $\cup_{i \in \omega} p\left(x, D_{i}, C H(D)\right)$ is inconsistent. Assume, in order to get a contradiction, that there is

$$
\vec{a}^{\prime} \models \cup_{i \in \omega} p\left(x, D_{i}, C H(D)\right)
$$

Since $\operatorname{tp}_{H}(\vec{a} D / C H(D))=\operatorname{tp}_{H}\left(\vec{a}^{\prime} D_{i} / C H(D)\right)$ and $\vec{a} \chi_{C H(M)} D H(M)$ we also get that $\vec{a}^{\prime} \ell_{C H(M)} D_{i} H(M)$.

This shows that $\vec{a}^{\prime}$ forks with each term in the independent sequence $\left\{D_{i} H(M)\right.$ : $i \in \omega\}$ over $C H(M)$, a contradiction to local character in $T$.

We just showed that $\vec{a} \chi_{C H(D)}^{\text {ind }} D$. On the other hand, if $\vec{a} \downarrow_{C}^{\text {ind }} D$, since $H(D) \subset D$ we also get $\vec{a} \downarrow_{C H(D)}^{\text {ind }} D$, a contradiction. Thus we get $\vec{a} \downarrow_{C}^{\text {ind }} D$ as we wanted.

Case 2: Assume that $\vec{a} \downarrow_{C H(M)} D H(M)$ and that $H B(\vec{a} / D) \neq H B(\vec{a} / C)$.

We will first prove:

Claim $H B(\vec{a} / D)$ is a proper subset of $H B(\vec{a} / C)$.

Write $H_{D}=H B(\vec{a} / D)$ and $H_{C}=H B(\vec{a} / C)$. Since $\vec{a} \downarrow_{C H_{C}} H$, from $\vec{a} \downarrow_{C H} D H$ we get $\vec{a} \downarrow_{C H_{C}} D H$ and so $\vec{a} \downarrow_{D H_{C}} H$. By minimality of $H$-basis, we have $H_{D} \subset$ $H_{C}$ and since the two sets are not equal we get the claim.

Let $H_{E}=H_{C} \backslash H_{D}, H_{E}$ is a set of independent elements over $C$. 
Assume, in order to get a contradiction, that $\operatorname{tp}_{H}(\vec{a} / D)$ does not fork over $C$. Then $\vec{a} \downarrow_{C}^{\text {ind }} D$. By Proposition 4.9, we get that $H_{E} \subset D$ and by Corollary 4.13 $H_{E} \subset \operatorname{acl}_{H}(\vec{a} C)$, so $H_{E} \downarrow_{C}^{\text {ind }} H_{E}$, a contradiction

We are interested in characterizing canonical bases. Note that we can work in $T^{e q}$, and thus, $\mathrm{acl}=\mathrm{acl}^{e q}$. We start with the following result which holds also in the geometric setting (with $e \in \operatorname{acl}^{e q}(B)$ ):

Lemma 5.4. Let $(M, H) \models T_{\mathcal{G}}^{\text {ind }}$ be sufficiently saturated, $B \subset M$ be an $H$ independent set, and $\vec{a} \in M, h=H B(\vec{a} / B)$. Suppose $e \in \operatorname{acl}^{\text {eq }}(B)$ (in the sense of $T)$ is such that $\vec{a} h \downarrow_{e} B$. Then $\vec{a} \downarrow_{e}^{\text {ind }} B$.

Proof. We use the characterization of forking given in Theorem 5.3

We first prove that $\vec{a} \downarrow_{\operatorname{acl}_{H}(e) H(M)} B H(M)$. Since $h=H B(\vec{a} / B)$, we have that $\vec{a} \downarrow_{B h} B H(M)$ and thus $\vec{a} \downarrow_{B e h} B H(M)$. By assumption $\vec{a} h \downarrow_{e} B$, so $\vec{a} \downarrow_{e h} B$ and by transitivity $\vec{a} \downarrow_{e h} B H(M)$ and we get $\vec{a} \downarrow_{e H(M)} B H(M)$ and $\vec{a} \downarrow_{\operatorname{acl}_{H}(e) H(M)} B H(M)$.

Now we prove that $H B(\vec{a} / B)=H B\left(\vec{a} / \operatorname{acl}_{H}(e)\right)$. Let $h_{0}=H B\left(\vec{a} / \operatorname{acl}_{H}(e)\right)$. We have $\vec{a} \downarrow_{B h} H(M)$ and since $e \in \operatorname{acl}^{e q}(B)$ and $\operatorname{acl}(B)=\operatorname{acl}_{H}(B)$ we get $\vec{a} \downarrow_{B \operatorname{acl}_{H}(e) h} H(M)$. But $\vec{a} \downarrow_{\operatorname{acl}_{H}(e) h} B$, so by transitivity we get $\vec{a} \downarrow_{\operatorname{acl}_{H}(e) h} H(M)$. This shows that $h \supset h_{0}$.

Since $\vec{a} h \downarrow_{\operatorname{acl}_{H}(e)} B$ we get $\vec{a} \downarrow_{\operatorname{acl}_{H}(e) h} B$ and by transitivity $\vec{a} \downarrow_{\operatorname{acl}_{H}(e) h} B H(M)$. On the other hand, $\vec{a} \downarrow_{\operatorname{acl}_{H}(e) h_{0}} H(M)$, so $\vec{a} \downarrow_{\operatorname{acl}_{H}(e) h_{0}} h$ and we get $\vec{a} \downarrow_{\operatorname{acl}_{H}(e) h_{0}} B H(M)$ so $\vec{a} \downarrow_{B h_{0}} H(M)$ and $h \subset h_{0}$.

Finally, the following result on canonical bases can be proved using a minor modification of the argument presented in [6]:

Proposition 5.5. Let $(M, H)$ be a sufficiently saturated $H$-structure of $T, B \subset M$ an $H$-independent set, and $\vec{a} \in M$. Then $C b_{H}(\vec{a} / B)$ and $\left.C b(\vec{a} H B(\vec{a} / B) / B)\right)$ are interalgebraic.

Proof. Let $e=C b(\vec{a} H B(\vec{a} / B) / B))$. We saw in the previous lemma that $\vec{a} \downarrow_{e}^{\text {ind }} B$ and thus $C b_{H}(\vec{a} / B) \in \operatorname{acl}_{H}^{e q}(e)$.

We will now prove that $e$ is in the algebraic closure of any Morley sequence in $\operatorname{stp}_{H}(\vec{a} / B)$.

Let $\left\{\vec{a}_{i}: i<\omega\right\}$ be an $\mathcal{L}_{H}$-Morley sequence in $\operatorname{tp}_{H}\left(\vec{a} / \operatorname{acl}_{H}^{e q}(B)\right)$. Let $h_{j}=$ $H B\left(\vec{a}_{j} / B\right)$ (viewed as an imaginary representing a finite set), so we have $h_{j} \in$ $\operatorname{dcl}_{H}\left(\vec{a}_{j} B\right)$. Thus $\left\{\vec{a}_{i} h_{i}: i<\omega\right\}$ is also an $\mathcal{L}_{H}$-Morley sequence over $B$.

Claim $\left\{\vec{a}_{i} h_{i}: i<\omega\right\}$ is also an $\mathcal{L}$-Morley sequence over $B$.

Since $\left\{\vec{a}_{i} h_{i}: i<\omega\right\}$ is an $\mathcal{L}_{H}$-Morley sequence over $B$, by Theorem 5.3 , we have $h_{j}=H B\left(\vec{a}_{j} / B \vec{a}_{<j} h_{<j}\right)$ and $\vec{a}_{j} h_{j} \downarrow_{B H(M)} \vec{a}_{<j} h_{<j} H(M)$. Since $\vec{a}_{j} h_{j} \downarrow_{B h_{j}} H(M)$ we get that $\vec{a}_{j} h_{j} \downarrow_{B h_{j}} \vec{a}_{<j} h_{<j}$. But $h_{j}=H B\left(\vec{a}_{j} / B \vec{a}_{<j} h_{<j}\right)$ and $B \vec{a}_{<j} h_{<j}$ is $H$ independent, so $B \vec{a}_{<j} h_{<j} \downarrow h_{j}$, and it follows that $\operatorname{tp}\left(\vec{a}_{j} h_{j} / B \vec{a}_{<j} h_{<j}\right)$ does not fork (in the sense of $\mathcal{L}$ ) over $B$. Thus, $\left\{\vec{a}_{i} h_{i}: i<\omega\right\}$ is a $\mathcal{L}$-Morley sequence in $\operatorname{tp}(\vec{a} h / B)$ over $B$.

By supersimplicity of $T$, we know that $e$ is in the definable closure of an initial segment of $\left\{\vec{a}_{i} h_{i}: i<\omega\right\}$. 
On the other hand, since $T_{\mathcal{G}}^{i n d}$ is supersimple there is $N \in \omega$ such that for all $n \geq N, \vec{a}_{n} \downarrow_{\vec{a}_{<N}}^{\text {ind }} B$. By Proposition $4.13 \operatorname{acl}_{H}\left(\vec{a}_{<N}\right)$ is $H$-independent. By Theorem 5.3 and the fact that $\left\{\vec{a}_{i}: i<\omega\right\}$ is a Morley sequence in $\operatorname{tp}_{H}\left(\vec{a} / \operatorname{acl}_{H}^{e q}(B)\right)$, $H B\left(\vec{a}_{n} / B\right)=H B\left(\vec{a}_{n} / \operatorname{acl}_{H}\left(B \vec{a}_{<N}\right)\right)=H B\left(\vec{a}_{n} / \operatorname{acl}_{H}\left(\vec{a}_{<N}\right)\right)$ and in particular $h_{n} \in$ $\operatorname{acl}_{H}\left(\vec{a}_{i}: i<\omega\right)$ for every $n \geq N$. We then get $e \in \operatorname{acl}_{H}^{e q}\left(\left\{\vec{a}_{i}: N \leq i<\omega\right\}\right)$ as we wanted.

Next, since $\left\{\vec{a}_{i}: i<\omega\right\}$ is a Morley sequence in $\operatorname{tp}_{H}\left(\vec{a} / \operatorname{acl}_{H}^{e q}(B)\right)$, we have

$$
\left\{\vec{a}_{i}: N \leq i<\omega\right\} \underset{C b_{H}(\vec{a} / B)}{\stackrel{i n d}{\perp}} B,
$$

and thus also

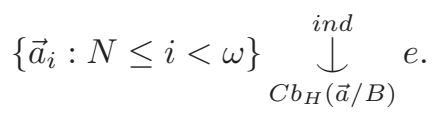

It follows that $e \in \operatorname{acl}_{H}^{e q}\left(C b_{H}(\vec{a} / B)\right)$, as needed.

Proposition 5.6. Assume that now that $T$ is also superstable. Then $T_{\mathcal{G}}^{\text {ind }}$ is superstable.

Proof. We already know that then $T_{\mathcal{G}}^{i n d}$ is supersimple. To check stability we use the criterion by Casanovas and Ziegler on expansions by predicates [9]. We already know by Proposition 4.4 that the induced structure on $H$ is stable. We also know that $T_{\mathcal{G}}^{i n d}$ is near model-complete, so every $\mathrm{cl}_{H}$-formula is equivalent to a boolean combination of formulas of the form $\exists x_{1} \in H \ldots \exists x_{m} \in H \varphi(\vec{x}, \vec{y})$, where $\varphi(\vec{x}, \vec{y})$ is an $\mathcal{L}$-formula. We can conclude that the theory $T_{\mathcal{G}}^{\text {ind }}$ is stable, thus also superstable.

\section{OnE-BASEDNESS}

An example of a one-based geometric theory $T$ such that $T^{\text {ind }}$ is not one-based was given in [5]. We follow the ideas on [8] to understand exactly when onebasedness is preserved.

We will focus our study on two "extreme" cases, on one hand we will study the case when $\mathcal{G}=" x=x$ " in any theory $T$. On the other hand we will consider the case when $T$ is a theory of U-rank $\omega^{\alpha}$ and the U-rank is continuous (see Section 3.4), in this case $\mathcal{G}$ is the union of the types of rank $\omega^{\alpha}$. We will see that, in both cases, one-basedness is preverved in $T_{\mathcal{G}}^{\text {ind }}$ whenever $T$ satisfies some kind of "triviality" with respect to the types in $\mathcal{G}$.

6.1. $\mathcal{G}=$ " $x=x^{\prime \prime}$. In this subsection we assume $T$ is supersimple, let $\mathcal{G}=" x=x^{\prime \prime}$ and assume that $T_{\mathcal{G}}^{i n d}$ is first order.

Definition 6.1. A theory $T$ is trivial (or trivial for freedom), if for every set $A$ and every tuples $\vec{a}, \vec{b}$, and $\vec{c}$ pairwise independent over $A$, we have that $\vec{a} \downarrow_{A} \vec{b} \vec{c}$.

Remark 6.2. The theory of the free pseudoplane (see example 3.3) is trivial.

Lemma 6.3 ([16], Proposition 8). Assume $T$ is one-based and trivial. If $\vec{a} \downarrow_{\vec{a}_{1}} \vec{b}_{1}$ and $\vec{a} \downarrow_{\vec{a}_{2}} \vec{b}_{2}$, then $\vec{a} \downarrow_{\vec{a}_{1} \vec{a}_{2}} \vec{b}_{1} \vec{b}_{2}$.

Proposition 6.4. Assume $T$ is a one-based theory and $\mathcal{G}=$ " $x=x "$, then $T_{\mathcal{G}}^{\text {ind }}$ is one-based if and only if $T$ is trivial. 
Proof. $(\Leftarrow)$ Assume $T$ is trivial. Let $\vec{a}$ be a tuple, $B$ an algebraically closed set in $(M, H)$ and $\vec{h}=H B(\vec{a})$. Since $\vec{a} \downarrow_{\vec{h}} H$ and $\vec{a} \downarrow_{B} B$, by the previous lemma we have that $\vec{a} \downarrow_{\vec{h} B} B H$. Therefore $H B(\vec{a} / B) \subset \vec{h}$ and

$$
\begin{aligned}
\operatorname{acl}_{H}^{e q}\left(C b_{H}(\vec{a} / B)\right) & =\operatorname{acl}_{H}^{e q}(C b(\vec{a} H B(\vec{a} / B) / B)) \\
& \subset \operatorname{acl}_{H}^{e q}(C b(\vec{a} \vec{h} / B)) \quad(\text { because } H B(\vec{a} / B) \subset \vec{h}) \\
& \subset \operatorname{acl}_{H}^{e q}\left(\operatorname{acl}^{e q}(\vec{a} \vec{h}) \cap \operatorname{acl}^{e q}(B)\right) \quad \text { (because } T \text { is one-based) } \\
& =\operatorname{acl}_{H}^{e q}(\vec{a}) \cap \operatorname{acl}_{H}^{e q}(B) .
\end{aligned}
$$

Thus, $T_{\mathcal{G}}^{\text {ind }}$ is one-based.

$(\Rightarrow)$ Assume $T_{\mathcal{G}}^{i n d}$ is one-based and $T$ is not trivial. Then, there are real tuples $\vec{a}, \vec{b}, \vec{h}$ and a set of parameters $A$ such that $\vec{a}, \vec{b}$ and $\vec{h}$ are pairwise independent but not independent over $A$ (from now on we will work over $A$ ). By the generalized extension property we can assume that $\vec{a} \downarrow H$. Also, we may assume $b$ and $h$ are singletons and, as $h \downarrow \vec{a}$, we may assume $h$ belongs to $H$ by the density property.

We may also assume that $b \downarrow_{\vec{a} h} H$ by the extension property.

Recall that $b \mathbb{X}_{\vec{a}} h$ and $h$ is a single element, then $h=H B(b / \vec{a})$.

If we take $\vec{a}^{\prime}=C b(b h / \vec{a})$ then the $\vec{a}^{\prime}, b$ and $h$ satisfy all the above properties, so we may take $\vec{a}=C b(b h / \vec{a})$.

By hypothesis $T_{\mathcal{G}}^{\text {ind }}$ is one-based, then $\operatorname{acl}_{H}^{e q}\left(C b_{H}(b / \vec{a})\right)=\operatorname{acl}_{H}^{e q}(b) \cap \operatorname{acl}_{H}^{e q}(\vec{a})$. Now, $\operatorname{acl}_{H}^{e q}(\vec{a})=\operatorname{acl}^{e q}(\vec{a})$ as $\vec{a} \downarrow H$. On the other hand, since $\vec{a} \downarrow H$ and $b \downarrow_{h \vec{a}} H$, we have that $b \underset{h}{\downarrow} H$. By hypothesis $b \downarrow h$ and, by transitivity, $b \downarrow H$. Hence

$$
H B(b)=\emptyset
$$

and

$$
\operatorname{acl}_{H}^{e q}(b)=\operatorname{acl}^{e q}(b)
$$

This means $\operatorname{acl}_{H}^{e q}\left(C b_{H}(b / \vec{a})\right)=\operatorname{acl}^{e q}(b) \cap \operatorname{acl}^{e q}(\vec{a})$.

However, recall that $\vec{a}=C b(b h / \vec{a})$, therefore

$$
\begin{aligned}
\operatorname{acl}^{e q}(\vec{a}) & =\operatorname{acl}_{H}^{e q}(\vec{a}) \\
& =\operatorname{acl}_{H}^{e q}(C b(b h / \vec{a})) \\
& =\operatorname{acl}_{H}^{e q}\left(C b_{H}(b / \vec{a})\right) \\
& =\operatorname{acl}^{e q}(b) \cap \operatorname{acl}^{e q}(\vec{a}) .
\end{aligned}
$$

This implies that $\vec{a} \subset \operatorname{acl}^{e q}(b)$, which yields a contradiction.

By a very well known result of Hrushovski, we now that if $T$ is stable, one-based and not trivial, then $T$ interprets an infinite group. Therefore we have the following corollary:

Corollary 6.5. Assume $T$ is stable. Then $T_{\mathcal{G}}^{\text {ind }}$ is one-based if and only if $T$ is one-based and does not interpret an infinite group. 
6.2. Theories of rank $\omega^{\alpha}$. In this subsection we will assume that $T$ is a theory of SU-rank $\omega^{\alpha}$ in which the $S U$-rank is continuous, we take $\mathcal{G}$ to be the union of all the types of rank $\omega^{\alpha}$ and we also assume that $T_{\mathcal{G}}^{i n d}$ is first order.

We can define a closure operator as $\operatorname{cl}(A)=\left\{x \mid S U(x / A)<\omega^{\alpha}\right\}$. It is not hard to check that cl defines a pregeometry (monotonicity comes from the Lascar inequality).

Definition 6.6. A pregeometry $(X, c l)$ is trivial if for every $A \subset X$,

$$
c l(A)=\bigcup_{a \in A} \operatorname{cl}(a) .
$$

Notice that if $G$ is a group of $S U$-rank $\omega^{\alpha}$ then cl is not trivial (take $a \downarrow b$ both of rank $\omega$ and $c=a+b$, then $c \in \operatorname{cl}(a, b) \backslash \operatorname{cl}(a) \cup \operatorname{cl}(b))$.

Remark 6.7. In the theory of the free pseudoplane (see example 3.3 ) the pregeometry generated by cl is trivial: for $A$ algebraically closed and a a single element, $S U(a / A)=d(a, A)$ where $d(a, A)$ is the minimum length of a path from a to an element of $A$ (or $\omega$ if there is no path). If $b \in \operatorname{cl}(A)$ it means that there is a path to some element $a \in A$ so $\operatorname{cl}(A)=\bigcup_{a \in A} \operatorname{cl}(a)$.

We will now prove that one-basedness is only preserved in $T_{\mathcal{G}}^{i n d}$ when the pregeometry cl is trivial. It is worth to notice that, unlike the $S U$-rank 1 case, the triviality of cl does not imply that $T$ is one-based. In fact, the theory of the free pseudoplane is the canonical example of a CM-trivial theory which is not onebased. This is the reason why the statement of the following proposition is a little bit different from the one from [8].

Lemma 6.8. If $\mathrm{cl}$ is trivial in $T$ then for every $\vec{a}$ and for every $B=\operatorname{acl}_{H}(B)$,

$$
H B(\vec{a} / B) \subset H B(\vec{a}) .
$$

Proof. Let $h=H B(\vec{a} / B)=\left\{h_{i} \mid i \in I\right\}$. By minimality of H-bases, for every $i \in I$ we have $\vec{a} \chi_{B h \backslash h_{i}} h_{i}$, then $h_{i} \in \operatorname{cl}\left(\vec{a} B h \backslash h_{i}\right)$. As $B$ is $H$-independent and $h_{i} \notin B$ then $h_{i} \downarrow B h \backslash h_{i}$, hence $h_{i} \notin \operatorname{cl}\left(B h \backslash h_{i}\right)$. By triviality it means that $h_{i} \in \operatorname{cl}\left(a_{i}\right)$ for some $a_{i} \in \vec{a}$. By exchange property $a_{i} \in \operatorname{cl}\left(h_{i}\right)$, this implies $a_{i}\left\lfloor h_{i}\right.$ and $a_{i} \downarrow_{h_{i}} H$ because $t p\left(a_{i} / h_{i}\right)$ is orthogonal to $H$, and $H$ consists of independent elements. We conclude that $h_{i}=H B\left(a_{i}\right)$ and

$$
H B(\vec{a} / B)=\left\{h_{i} \mid i \in I\right\}=\bigcup_{a_{i} \in A} H B\left(a_{i}\right) \subset H B(\vec{a})
$$

Proposition 6.9. Assume $T$ is one-based, then $T_{\mathcal{G}}^{i n d}$ is one-based if and only if $\mathrm{cl}$ is trivial in $T$.

Proof. $(\Leftarrow)$ Assume cl is trivial, let $\vec{a}$ be a tuple, $B$ an algebraic closed set in $(M, H)$ and $\vec{h}=H B(\vec{a} / B)$. By the characterization of canonical bases, acl ${ }^{\mathrm{eq}}{ }_{H}\left(C b_{H}(\vec{a} / B)\right)=$ $\operatorname{acl}^{\text {eq }}(C b(\vec{a} \vec{h} / B))$, as $T$ is one-based, $C b(\vec{a} \vec{h} / B) \subset \operatorname{acl}^{\text {eq }}(\vec{a} \vec{h})$. By the previous lemma, $\vec{h} \subset H B(\vec{a})$, and, thus, $C b_{H}(\vec{a} / B) \subset \operatorname{acl}_{H}^{\mathrm{eq}}(\vec{a} H B(\vec{a}))=\operatorname{acl}_{H}^{\mathrm{eq}}(\vec{a})$, i.e. $T_{\mathcal{G}}^{\text {ind }}$ is one-based.

$(\Rightarrow)$ Assume $T_{\mathcal{G}}^{i n d}$ is one-based and cl is not trivial, then there are a tuple $\vec{a}$ and elements $b$ and $h$ such that $b \in \operatorname{cl}(\vec{a} h)$ and $b \notin \operatorname{cl}(\vec{a}) \cup \operatorname{cl}(h)$. Since $b \notin \operatorname{cl}(\vec{a})$ then 
$h \notin \operatorname{cl}(\vec{a})$, therefore

$$
S U(b)=S U(h)=\omega^{\alpha} .
$$

We can take $\vec{a}$ a cl independent tuple minimal with this property and, by the generalized extension property, we may assume that $\vec{a} \downarrow H$. Moreover, as $h \notin$ $\operatorname{cl}(\vec{a})$, we may assume also that $h$ belongs to $H$ by the density property.

As $b \in \operatorname{cl}(\vec{a} h)$, we have that $t p(b / \vec{a} h)$ is orthogonal to $H$, therefore $b \downarrow_{h \vec{a}} H$. Recall that $b \mathbb{Z}_{\vec{a}} h$ and $h$ is a single element, and, thus, $h=H B(b / \vec{a})$. By hypothesis $T_{\mathcal{G}}^{\text {ind }}$ is one-based, then $\operatorname{acl}_{H}{ }^{\mathrm{eq}}\left(C b_{H}(b / \vec{a})\right)=\operatorname{acl}_{H} \mathrm{eq}_{H}(b) \cap \operatorname{acl}_{H}^{\mathrm{eq}}(\vec{a})$. Now, $\operatorname{acl}^{\mathrm{eq}}(\vec{a})=\operatorname{acl}^{\mathrm{eq}}(\vec{a})$ as $\vec{a} \downarrow H$. On the other hand, as $\vec{a} \downarrow H$, and $b \downarrow_{h \vec{a}} H$ we have $b \underset{h}{\downarrow} H$. By hypothesis $b \notin \operatorname{cl}(h)$, hence $b \downarrow h$ (recall that $b$ is a single element) and by transitivity $b \downarrow H$. So $H B(b)=\emptyset$ and $\operatorname{acl}^{\mathrm{eq}} H(b)=\operatorname{acl}^{\mathrm{eq}}(b)$. This means $\operatorname{acl}_{H}^{\mathrm{eq}}\left(C b_{H}(b / \vec{a})\right)=\operatorname{acl}^{\mathrm{eq}}(b) \cap \operatorname{acl}^{\mathrm{eq}}(\vec{a}) . \mathrm{But} \mathrm{acl}_{H}^{\mathrm{eq}}\left(C b_{H}(b / \vec{a})\right)=\operatorname{acl}_{H}^{\mathrm{eq}}(C b(b h / \vec{a}))$, so, in particular, we have that

$$
b \notin \operatorname{cl}(C b(b h / \vec{a})) .
$$

Now, since $b h \downarrow_{C b(b h / \vec{a})} a$, we have that $b \downarrow_{h C b(b h / \vec{a})} h \vec{a}$, therefore

$$
b \in \operatorname{cl}(h C b(b h / \vec{a}))
$$

so any maximal cl-independent subset $\vec{d}$ of $\operatorname{cl}(C b(b h / \vec{a}))$ satisfies that

$$
b \in \operatorname{cl}(\overrightarrow{d h})
$$

and

$$
b \notin \operatorname{cl}(\vec{d}) \cup \operatorname{cl}(h)
$$

The minimality of the length of $\vec{a}$ yields $\operatorname{cl}(C b(b h / \vec{a}))=\operatorname{cl}(\vec{a})$, hence $\operatorname{cl}(\vec{a})=$ $\operatorname{cl}\left(\operatorname{acl}^{\mathrm{eq}}(\vec{a}) \cap \operatorname{acl}^{\mathrm{eq}}(b)\right) \subset \operatorname{cl}(\vec{a}) \cap \operatorname{cl}(b)$, then $\vec{a} \in \operatorname{cl}(b)$ and $h \in \operatorname{cl}(\vec{a} b) \subset \operatorname{cl}(b)$. This is a contradiction.

\section{Ampleness}

The notion of ampleness, defined by Pillay, captures forking complexity. $\mathrm{He}$ proved in [17] that a theory $T$ is one-based if and only if is not 1-ample, a theory $T$ is CM-trivial if and only if is not 2-ample. Moreover if $T$ interprets a field then it is $n$-ample for every $n$. We know that non-1-ampleness (one-basedness) is not always preserved in $T_{\mathcal{G}}^{i n d}$. In contrast, we show in this section that, for $n \geq 2$, non- $n$-ampleness is preserved in the expansion.

Note that, in contrast with the previous section, we do not require any conditions on $\mathcal{G}$.

Definition 7.1. A supersimple theory $T$ is $n$-ample if (possibly after naming some parameters) there exist tuples $a_{0}, \ldots, a_{n}$ in $M$ satisfying the following conditions:

For all $1 \leq i \leq n-1$.

(1) $a_{i+1} \downarrow a_{i-1} \ldots a_{0}$,

(2) $\operatorname{acl}^{e q}\left(a_{0} \ldots a_{i-1} a_{i+1}\right) \cap \operatorname{acl}^{e q}\left(a_{0} \ldots a_{i-1} a_{i}\right)=\operatorname{acl}^{e q}\left(a_{0} \ldots a_{i-1}\right)$.

(3) $a_{n} \underset{\operatorname{acl}^{e q}\left(a_{1}\right) \cap \operatorname{acl}^{e q}\left(a_{0}\right)}{\not} a_{0}$. 
The tuple $a_{0}, \ldots, a_{n}$ is $n$-ample if it satisfies the above properties.

Following [8] we prove that, for $n \geq 2$, non- $n$-ampleness is preserved in $T_{\mathcal{G}}^{\text {ind }}$. First we need the following lemmas.

Lemma 7.2 (15] Fact 2.4). If $A, B, C$ and $D$ are algebraically closed such that $A \cap B=C$, and $A B \downarrow_{C} D$, then $\operatorname{acl}^{e q}(A D) \cap \operatorname{acl}^{e q}(B D)=\operatorname{acl}^{e q}(C D)$.

Proof. Let $c \in \operatorname{acl}^{e q}(A D) \cap \operatorname{acl}^{e q}(B D)$. Since $D \downarrow_{C} A B$ then $A D \downarrow_{A} A B$ and $c D \downarrow_{A} A B$. Therefore

$$
C b(c D / A B) \subset A .
$$

In the same way we obtain that

$$
C b(c D / A B) \subset B .
$$

Therefore

$$
C b(c D / A B) \subset A \cap B=C,
$$

so

$$
c D \underset{C}{\downarrow} A B
$$

This implies that $c \in \operatorname{acl}^{e q}(D C)$.

Lemma 7.3. A theory is $n$-ample if and only if there exist tuples $a_{0}, \ldots, a_{n}$ in $M^{e q}$ satisfying the following conditions:

(1) $a_{i+1} \downarrow a_{i-1} \ldots a_{0}$ for all $i \leq n-1$,

(2) $\operatorname{acl}^{e q}\left(a_{i-1} a_{i+1}\right) \cap \operatorname{acl}^{e q}\left(a_{i-1} a_{i}\right)=\operatorname{acl}^{e q}\left(a_{i-1}\right)$ for all $i \leq n-2$.

(3) $a_{n} \underset{\operatorname{acl}^{e q}\left(a_{1}\right) \cap \operatorname{\cap acl}^{e q}\left(a_{0}\right)}{\chi} a_{0}$.

Proof. $(\Rightarrow)$ Assume $T$ is n-ample and let $a_{0}, \ldots, a_{n}$ be the ample tuple. Then $\operatorname{acl}^{e q}\left(a_{i-1} a_{i+1}\right) \cap \operatorname{acl}^{e q}\left(a_{i-1} a_{i}\right) \subset \operatorname{acl}^{e q}\left(a_{0}, \ldots, a_{i-1}\right) \cap \operatorname{acl}^{e q}\left(a_{i-1} a_{i}\right)$.

Since $a_{i} \downarrow_{a_{i-1}} a_{0} \ldots a_{i-2}$, this intersection is contained in $\operatorname{acl}^{e q}\left(a_{i-1}\right)$.

$(\Leftarrow)$ Assume $a_{0}, \ldots, a_{n}$ satisfy the above conditions, then using the previous lemma with $A=\operatorname{acl}^{e q}\left(a_{i-1} a_{i+1}\right), B=\operatorname{acl}^{e q}\left(a_{i-1} a_{i}\right), C=\operatorname{acl}^{e q}\left(a_{i-1}\right)$ and $D=\operatorname{acl}^{e q}\left(a_{0}, \ldots, a_{i-2}\right)$, we get that

$$
\operatorname{acl}^{e q}\left(a_{0} \ldots a_{i-1} a_{i+1}\right) \cap \operatorname{acl}^{e q}\left(a_{0} \ldots a_{i-1} a_{i}\right)=\operatorname{acl}^{e q}\left(a_{0} \ldots a_{i-1}\right) .
$$

i.e. $a_{0}, \ldots, a_{n}$ is $n$-ample.

We will use the above characterization of ampleness to prove the main result of this section (Theorem 7.5).

Lemma 7.4. Let $A \subset B, A=\operatorname{acl}_{H}^{e q}(A)$ and $B=\operatorname{acl}_{H}^{e q}(B)$. If $\operatorname{acl}_{H}^{e q}(c A) \cap B=A$ then $H B(c / A) \subset H B(c / B)$.

Proof. By Lemma 4.9 we have

$$
H B(c / A) \subset H B(c / B) \cup H B(B) .
$$

Now, if $H B(c / A) \cap H(B)=\emptyset$ we are done, but

$$
H B(c / A) \cap H B(B) \subset \operatorname{acl}_{H}^{e q}(c A) \cap B=A
$$

and $H B(c / A) \cap A=\emptyset$. 
Theorem 7.5. For $n \geq 2$, the theory $T$ is $n$-ample if and only if $T_{\mathcal{G}}^{\text {ind }}$ is.

Before doing the proof, recall that the canonical bases in $T_{\mathcal{G}}^{\text {ind }}$ are interalgebraic with canonical bases of $T$, therefore every "new" imaginary element is interalgebraic with an "old" imaginary. By virtue of Lemma 4.12 we may assume that $\operatorname{acl}_{H}^{e q}(A)=$ $\operatorname{acl}^{\mathrm{eq}}(A H B(A))$.

Proof. Assume that $T$ is $n$-ample. Let $a_{0}, \ldots, a_{n}$ be such that

(1) $\operatorname{acl}^{e q}\left(a_{i} a_{i+1}\right) \cap \operatorname{acl}^{e q}\left(a_{i} a_{i+2}\right)=\operatorname{acl}^{e q}\left(a_{i}\right)$ for all $i<n-1$,

(2) $a_{i+1} \downarrow_{a_{i}} a_{0} \ldots a_{i-1}$ for all $i<n$,

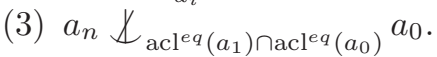

By the generalized extension property, we may assume that $a_{0} \ldots a_{n} \downarrow H$, hence $H B(X)=\emptyset$ for every $X \subset\left\{a_{0}, \ldots, a_{n}\right\}$, which implies that $\operatorname{acl}^{e q}(X)=\operatorname{acl}_{H}^{e q}(X)$ for $X \subset\left\{a_{0}, \ldots, a_{n}\right\}$. Therefore

$$
\begin{aligned}
\operatorname{acl}_{H}^{e q}\left(a_{i} a_{i+1}\right) \cap \operatorname{acl}_{H}^{e q}\left(a_{i} a_{i+2}\right) & =\operatorname{acl}^{e q}\left(a_{i} a_{i+1}\right) \cap \operatorname{acl}^{e q}\left(a_{i} a_{i+2}\right) \\
& =\operatorname{acl}^{e q}\left(a_{i}\right) \\
& =\operatorname{acl}_{H}^{e q}\left(a_{i}\right) .
\end{aligned}
$$

On the other hand, we have

$$
a_{i+1} \underset{a_{i}}{\stackrel{\text { ind }}{\downarrow}} a_{0} \ldots a_{i-1}
$$

because

$$
a_{i+1} \underset{a_{i}}{\downarrow} a_{0} \ldots a_{i-1}
$$

and $H B\left(a_{i+1} / a_{0} \ldots a_{i}\right)=\emptyset$.

Finally, since $a_{n} \mathbb{X}_{\mathrm{acl}^{e q}\left(a_{1}\right) \cap \operatorname{acl}^{e q}\left(a_{0}\right)} a_{0}$, we have that $a_{n} \mathbb{Z}_{\mathrm{acl}^{l e q}\left(a_{1}\right) \cap \operatorname{acl}^{e q}\left(a_{0}\right)}^{\text {ind }} a_{0}$. Thus, the tuple $a_{0}, \ldots a_{n}$ is n-ample in the sense of $T_{\mathcal{G}}^{\text {ind }}$.

Assume now that $T$ is not $n$-ample, let $a_{0}, \ldots, a_{n} \in \mathfrak{C}$ be tuples such that:

(1) $\operatorname{acl}_{H}^{e q}\left(a_{i} a_{i+1}\right) \cap \operatorname{acl}_{H}^{e q}\left(a_{i} a_{i+2}\right)=\operatorname{acl}_{H}^{e q}\left(a_{i}\right)$ for all $i<n$.

(2) $a_{i+1} \downarrow_{a_{i}}^{i n d} a_{0} \ldots a_{i-1}$ for all $i<n$.

We may assume also that $\operatorname{acl}_{H}^{e q}\left(a_{i}\right)=\operatorname{acl}^{e q}\left(a_{i}\right)$ for every $i$.

Consider $h_{i}=H B\left(a_{n} / a_{i}\right)$. We claim that $h_{0} \subset h_{1} \subset \cdots \subset h_{n-1}$.

Proof of the claim. Since $a_{n} \downarrow_{a_{i+2}}^{\text {ind }} a_{i} a_{i+1}$, we have that $a_{n} a_{i} \downarrow_{a_{i} a_{i+2}}^{\text {ind }} a_{i} a_{i+1}$. Hence

$$
\begin{aligned}
\operatorname{acl}_{H}^{e q}\left(a_{n} a_{i}\right) \cap \operatorname{acl}_{H}^{e q}\left(a_{i} a_{i+1}\right) & \subset \operatorname{acl}_{H}^{e q}\left(a_{i} a_{i+2}\right) \cap \operatorname{acl}_{H}^{e q}\left(a_{i} a_{i+1}\right) \\
& \subset \operatorname{acl}_{H}^{e q}\left(a_{i}\right) \text { by assumption }(1)
\end{aligned}
$$

Using Lemma 7.4 (by making $A=a_{i}, B=a_{i} a_{i+1}$ and $c=a_{n}$ ) we conclude that $h_{i}=H B(c / A) \subset H B(c / B)=H B\left(a_{n} / a_{i} a_{i+1}\right)$. But $a_{n} \downarrow_{a_{i+1}}^{i n d} a_{i}$, hence $H B\left(a_{n} / a_{i} a_{i+1}\right)=H B\left(a_{n} / a_{i+1}\right)=h_{i+1}$. 
In particular $h_{0} \subset h_{n-1}$.

Notice that

$$
h_{0} \subset h_{n-2} \subset \operatorname{acl}_{H}^{e q}\left(a_{n-2} a_{n}\right),
$$

hence

$$
\operatorname{acl}^{e q}\left(a_{n-2} a_{n} h_{0}\right) \subset \operatorname{acl}_{H}^{e q}\left(a_{n-2} a_{n}\right)
$$

and

$$
\begin{aligned}
\operatorname{acl}^{e q}\left(a_{n-2} a_{n-1}\right) \cap \operatorname{acl}^{e q}\left(a_{n-2} a_{n} h_{0}\right) & \subset \operatorname{acl}_{H}^{e q}\left(a_{n-2} a_{n-1}\right) \cap \operatorname{acl}_{H}^{e q}\left(a_{n-2} a_{n}\right) \\
& =\operatorname{acl}_{H}^{\text {eq }}\left(a_{n-2}\right) .
\end{aligned}
$$

On the other hand,

$$
a_{n} h_{n-1} \stackrel{\text { ind }}{\downarrow} a_{0} \ldots a_{n-1},
$$

so since $a_{n} a_{n-1} h_{n-1}$ is $H$-independent

$$
a_{n} h_{n-1} \underset{a_{n-1}}{\downarrow} a_{0} \ldots a_{n-1},
$$

and

$$
\left.a_{n} h_{0} \underset{a_{n-1}}{\downarrow} a_{0} \ldots a_{n-1} \text { (because } h_{0} \subset h_{n-1}\right),
$$

then, setting $a_{n}^{\prime}=a_{n} h_{0}$ and $a_{i}^{\prime}=a_{i}$ for $i<n$, we have that:

(1) $\operatorname{acl}^{e q}\left(a_{i}^{\prime} a_{i+1}^{\prime}\right) \cap \operatorname{acl}^{e q}\left(a_{i}^{\prime} a_{i+2}^{\prime}\right)=\operatorname{acl}^{e q}\left(a_{i}^{\prime}\right)$ for all $i<n$.

(2) $a_{i+1}^{\prime} \downarrow_{a_{i}^{\prime}} a_{0}^{\prime} \ldots a_{i-1}^{\prime}$ for all $i<n$.

Since $T$ is not $n$-ample, it follows that

$$
a_{n}^{\prime} \underset{\operatorname{acl}^{e q}\left(a_{0}^{\prime}\right) \cap \operatorname{aacl}^{e q}\left(a_{1}^{\prime}\right)}{\perp} a_{0}^{\prime}
$$

i.e.

$$
a_{n} h_{0} \underset{\operatorname{acl}^{e q}\left(a_{0}\right) \cap \operatorname{acl}^{e q}\left(a_{1}\right)}{\downarrow} a_{0} .
$$

As $h_{0}=H B\left(a_{n} / a_{0}\right)$, by the characterization of canonical bases we have that $C b\left(a_{n} h_{0} / a_{0}\right)$ is interalgebraic with $C b_{H}\left(a_{n} / a_{0}\right)$; this implies that

$$
a_{n} \underset{\operatorname{acl}_{H}^{e q}\left(a_{0}\right) \cap \operatorname{acl}_{H}^{e q}\left(a_{1}\right)}{\stackrel{i n d}{\downarrow}} a_{0} .
$$

Thus, $T_{\mathcal{G}}^{\text {ind }}$ is not $n$-ample.

\section{Geometry modulo $H$ in the one-Based Case}

In this section we consider the case when $T$ is one-based of SU-rank $\omega^{\alpha}$ in which SU-rank is continuous, and follow the proofs of Theorem 5.13 [20] and the results of Section 6 of [20], and Section 4 of [5], to study the geometry induced by cl localized at $H(M)$. Many of the proofs are nearly identical to the ones from [20] and [5], we include them for completeness. As before, we take $\mathcal{G}$ to be a type of SU-rank $\omega^{\alpha}$.

Let $(M, H)$ be a sufficiently saturated model of $T_{\mathcal{G}}^{\text {ind }}$. Let $\mathrm{cl}_{H}$ be the localization of the operator cl at $H(M)$, i.e. $\operatorname{cl}_{H}(A)=\operatorname{cl}(A \cup H(M))$. Thus, $a \in \operatorname{cl}_{H}(B)$ means $S U(a / B \cup H(M))<\omega^{\alpha}$. 
Proposition 8.1. Suppose $T$ is one-based. Then the pregeometry $\left(M, \mathrm{cl}_{H}\right)$ is modular.

Proof. It suffices to show that for any $a, b \in M$ and a small set $C \subset M$, if $a \in$ $\mathrm{cl}_{H}(b C)$ then there exists $d \in \mathrm{cl}_{H}(C)$ such that $a \in \mathrm{cl}_{H}(b d)$. We may assume that $a, b \notin \mathrm{cl}_{H}(C)$. Thus, $S U(a / C H(M))=S U(b / C H(M))=\omega^{\alpha}$.

Let $\vec{h} \in H(M)$ be finite such that $a \in \operatorname{cl}(b C \vec{h})$. Let $e=C b(a b / C \vec{h})$. Thus, by one-basedness of $T, e \in \operatorname{acl}^{e q}(a b) \cap \operatorname{acl}^{e q}(C \vec{h})$. By the density property, there is $b^{\prime} \models \operatorname{tp}\left(b / \operatorname{acl}^{e q}(C \vec{h})\right), b^{\prime} \in H(M)$. Take $a^{\prime} \in M$ such that $\operatorname{tp}\left(a^{\prime} b^{\prime} / \operatorname{acl}^{e q}(C \vec{h})\right)=$ $\operatorname{tp}\left(a b / \operatorname{acl}^{e q}(C \vec{h})\right)$. Then $e \in \operatorname{acl}^{e q}\left(a^{\prime} b^{\prime}\right)$. Clearly, $a^{\prime} \in \operatorname{cl}\left(b^{\prime} C \vec{h}\right) \subset \operatorname{cl}_{H}(C)$. Also, $a b \downarrow_{e} C \vec{h}$ implies $S U(a / b e)=S U(a / b C \vec{h})<\omega^{\alpha}$. Since $e \in \operatorname{acl}^{e q}\left(a^{\prime} b^{\prime}\right)$, we have $S U\left(a / b a^{\prime} b^{\prime}\right) \leq S U(a / b e)<\omega^{\alpha}$. Since $b^{\prime} \in H(M)$, this implies $a \in \mathrm{cl}_{H}\left(b a^{\prime}\right)$. Hence, taking $d=a^{\prime}$, we have $d \in \operatorname{cl}_{H}(C)$ and $a \in \mathrm{cl}_{H}(b d)$, as needed.

Let $\left(M^{*}, \mathrm{cl}^{*}\right)$ be the geometry associated with $\left(M, \mathrm{cl}_{H}\right)$ (i.e. $M^{*}$ is the set $M \backslash \operatorname{cl}_{H}(\emptyset)$ modulo the relation $\left.\operatorname{cl}_{H}(x)=\operatorname{cl}_{H}(y)\right)$. For any $a \notin \mathrm{cl}_{H}(\emptyset)$, let $a^{*}$ be the class of $a$ modulo the relation $\operatorname{cl}_{H}(x)=\mathrm{cl}_{H}(y)$. Define the relation $\sim$ by

$$
a^{*} \sim b^{*} \Longleftrightarrow\left|\mathrm{cl}^{*}\left(a^{*}, b^{*}\right)\right| \geq 3 \text { or } a^{*}=b^{*} .
$$

Lemma 8.2. For any $a, b \in M, a^{*} \sim b^{*}$ if and only if there exist $d_{1}, \ldots, d_{n} \in M$ such that

$$
a^{*} \in \mathrm{cl}^{*}\left(b^{*} d_{1}^{*} \ldots d_{n}^{*}\right) \backslash \mathrm{cl}^{*}\left(d_{1}^{*} \ldots d_{n}^{*}\right) .
$$

Proof. The "only if" direction is clear. For the "if" direction, suppose $a^{*} \neq b^{*}$ and $a^{*} \in \mathrm{cl}^{*}\left(b^{*} d_{1}^{*} \ldots d_{n}^{*}\right) \backslash \mathrm{cl}^{*}\left(d_{1}^{*} \ldots d_{n}^{*}\right)$. We may assume that $n \geq 1$ is minimal such. Then $a \in \operatorname{cl}\left(b d_{1} \ldots d_{n} h_{1} \ldots h_{k}\right)$ for some $h_{1}, \ldots, h_{k} \in H(M)$. We may assume that $k$ is minimal such. Then the tuple $a b d_{2} \ldots d_{n} h_{1} \ldots h_{k}$ is cl-independent. By the density property, we can find $d_{2}^{\prime}, \ldots, d_{n}^{\prime} \in H(M)$ such that $\operatorname{tp}\left(d_{2}^{\prime}, \ldots, d_{n}^{\prime} / a b \vec{h}\right)=$ $\operatorname{tp}\left(d_{2}, \ldots, d_{n} / a b \vec{h}\right)$. Let $d_{1}^{\prime} \in M$ be such that

$$
\operatorname{tp}\left(d_{1}^{\prime}, d_{2}^{\prime}, \ldots, d_{n}^{\prime} / a b \vec{h}\right)=\operatorname{tp}\left(d_{1}, d_{2}, \ldots, d_{n} / a b \vec{h}\right) .
$$

Then $d_{1}^{\prime} \notin \operatorname{cl}_{H}(\emptyset)$ and $\left(d_{1}^{\prime}\right)^{*} \in \operatorname{cl}^{*}\left(a^{*}, b^{*}\right)$, while $\left(d_{1}^{\prime}\right)^{*} \neq a^{*}, b^{*}$. Thus, $\left|\operatorname{cl}^{*}\left(a^{*}, b^{*}\right)\right| \geq$ 3 , as needed.

Lemma 8.3. The relation $\sim$ is an equivalence on $M^{*}$.

Proof. Reflexivity and symmetry are clear. For transitivity, assume $a^{*} \sim b^{*} \sim$ $c^{*}$, with all three distinct. Then there exist $d_{1}^{*} \in \operatorname{cl}^{*}\left(a^{*} b^{*}\right) \backslash\left\{a^{*}, b^{*}\right\}$ and $d_{2}^{*} \in$ $\mathrm{cl}^{*}\left(b^{*}, c^{*}\right) \backslash\left\{b^{*}, c^{*}\right\}$. If $d_{1}^{*}=d_{2}^{*}$, then $c^{*} \in \operatorname{cl}^{*}\left(b^{*}, d_{2}^{*}\right)=\mathrm{cl}^{*}\left(b^{*}, d_{1}^{*}\right)=\operatorname{cl}^{*}\left(a^{*}, d_{1}^{*}\right)$, and therefore $d_{1}^{*}=d_{2}^{*} \in \mathrm{cl}^{*}\left(a^{*}, c^{*}\right) \backslash\left\{a^{*}, c^{*}\right\}$, hence $a^{*} \sim c^{*}$.

Now, assume that $d_{1}^{*} \neq d_{2}^{*}$ and $a^{*} \in \operatorname{cl}^{*}\left(d_{1}^{*}, d_{2}^{*}\right)$. If $a^{*}=d_{2}^{*}$, then $b^{*}$ witnesses $a^{*} \sim c^{*}$. If $a^{*} \neq d_{2}^{*}$, then $d_{2}^{*} \in \operatorname{cl}^{*}\left(a^{*}, d_{1}^{*}\right)$. We also have $b^{*} \in \operatorname{cl}^{*}\left(a^{*}, d_{1}^{*}\right), c^{*} \in$ $\mathrm{cl}^{*}\left(b^{*}, d_{2}^{*}\right)$. Thus, $c^{*} \in \mathrm{cl}^{*}\left(a^{*}, d_{1}^{*}\right)$. If $c^{*}=d_{1}^{*}, b^{*}$ witnesses $a^{*} \sim c^{*}$. If $c^{*} \neq d_{1}^{*}$, then $d_{1}^{*}$ witnesses $a^{*} \sim c^{*}$. Finally, assume that $d_{1}^{*} \neq d_{2}^{*}$ and neither $a^{*} \notin \operatorname{cl}^{*}\left(d_{1}^{*}, d_{2}^{*}\right)$. Then

$$
a^{*} \in \mathrm{cl}^{*}\left(c^{*} d_{1} d_{2}^{*}\right) \backslash \mathrm{cl}^{*}\left(d_{1}^{*} d_{2}^{*}\right) .
$$

Thus, by Lemma 8.2, $a^{*} \sim c^{*}$. 
For any $a^{*} \in M^{*}$ let $\left[a^{*}\right]$ denote the $\sim$-class of $a^{*}$.

Lemma 8.4. The $\sim$-classes are closed in the sense of $\mathrm{cl}^{*}$, i.e. for any $a^{*} \in M^{*}$, we have $\mathrm{cl}^{*}\left(\left[a^{*}\right]\right)=\left[a^{*}\right]$.

Proof. Assume $c^{*} \in \mathrm{cl}^{*}\left(b_{1}^{*}, \ldots, b_{n}^{*}\right), \vec{b}^{*}=\left(b_{1}^{*}, \ldots, b_{n}^{*}\right) \in\left[a^{*}\right]$ minimal such tuple, and $n>1$ (if $n=1$, we have $c^{*}=b_{1}^{*}$ ). Then $b_{1}^{*} \ldots b_{n-1}^{*}$ witnesses $c^{*} \sim b_{n}^{*}$, by Lemma 8.2 .

For any geometry $(X, C l)$, a non-empty subset of $X$, with the closure operator induced by $C l$, is referred to as a subgeometry of $(X, C l)$. Clearly, a subgeometry is itself a geometry. Next lemma shows that $\sim \operatorname{splits}\left(M^{*}, \mathrm{cl}^{*}\right)$ into disjoint subgeometries of the form $\left(\left[a^{*}\right], \mathrm{cl}^{*}\right)$, with no "interaction" between them.

Lemma 8.5. For any $A \subset M^{*}, \mathrm{cl}^{*}(A)=\bigcup_{\left[a^{*}\right] \in M^{*} / \sim} \mathrm{cl}^{*}\left(A \cap\left[a^{*}\right]\right)$.

Proof. Suppose $c^{*} \in \mathrm{cl}^{*}(A)$, and $a_{1}^{*}, \ldots, a_{n}^{*} \in A$ is a tuple such that $c \in \operatorname{cl}^{*}\left(a_{1}^{*}, \ldots, a_{n}^{*}\right)$, and $n$ is minimal such. It suffices to show that $a_{i}^{*}$ all come from the same $\sim$-class. If $n=1$, we are done. Suppose $n>1$. Then $c^{*} a_{3}^{*} \ldots a_{n}^{*}$ witnesses $a_{1}^{*} \sim a_{2}^{*}$ by Lemma 8.2 Similarly, $a_{1}^{*} \sim a_{i}^{*}$ for all $2<i \leq n$. Thus, all $a_{i}^{*}$ belong to the same $\sim$-class.

Next, we will show that the $\sim$-classes are either singletons or infinite dimensional (as geometries).

Lemma 8.6. If $\left|\left[a^{*}\right]\right|>1$, then $\operatorname{dim}\left(\left[a^{*}\right]\right)$ is infinite.

Proof. Suppose there exists $b^{*} \sim a^{*}, b^{*} \neq a^{*}$. Let $c^{*} \in \mathrm{cl}^{*}\left(a^{*}, b^{*}\right) \backslash\left\{a^{*}, b^{*}\right\}$. Let $a, b, c \in M$ be representatives of the classes $a^{*}, b^{*}$ and $c^{*}$ modulo the relation $\operatorname{cl}_{H}(x)=\operatorname{cl}_{H}(y)$, respectively.

Then $S U(a / H(M))=S U(b / a H(M))=\omega$. By the extension property, we can find $b_{i} \models \operatorname{tp}(b / a), i \in \omega$, independent over $a H(M)$. Then, by Lemma 2.7 $\operatorname{tp}_{H}\left(b_{i} / a\right)=\operatorname{tp}_{H}(b / a)$ for all $i \in \omega$. Also, $b_{i}$ are $\operatorname{cl}_{H}$-independent over $a$. Let $c_{i}$ be such that $\operatorname{tp}_{H}\left(b_{i} c_{i} / a\right)=\operatorname{tp}_{H}(b c / a)$ for $i \in \omega$. Passing to the geometry $\left(M^{*}, \mathrm{cl}^{*}\right)$, we get $b_{i}^{*} \sim a^{*}$ witnessed by $c_{i}^{*}, i \in \omega$, with $b_{i} \mathrm{cl}^{*}$-independent over $a^{*}$. This shows that that $\left(\left[a^{*}\right], \mathrm{cl}^{*}\right)$ is infinite dimensional.

Recall the following classical fact (see [13]) about projective geometries.

Fact 8.7. A non-trivial modular geometry of dimension $\geq 4$ in which any closed set of dimension 2 has size $\geq 3$ is a projective geometry over some division ring.

Lemma 8.8. If $T$ is one-based and $\left|\left[a^{*}\right]\right|>1$, the geometry $\left(\left[a^{*}\right], c l\right)$ is an infinite dimensional projective geometry over some division ring.

Proof. By Proposition 8.1. $\left(M^{*}, \mathrm{cl}^{*}\right)$ is modular. By Lemma 8.5. $\left[a^{*}\right]$ is closed in $\left(M^{*}, \mathrm{cl}^{*}\right)$, and hence $\left(\left[a^{*}\right], \mathrm{cl}^{*}\right)$ is also modular. Since $\left|\left[a^{*}\right]\right|>1,\left(\left[a^{*}\right], \mathrm{cl}^{*}\right)$ is nontrivial (there are two distinct point having a third one in its closure). Thus, the statement follows by Fact 8.7 and the definition of $\sim$.

We are now ready to prove the characterization of the geometry of $\mathrm{cl}_{H}$, as well as the original geometry of $\mathrm{cl}$ in the case when $T$ is one-based. 
Proposition 8.9. Suppose $T$ is a one-based supersimple theory of $S U$-rank $\omega$, $(N, H)$ a sufficiently (e.g. $\left.|T|^{+}-\right)$saturated models of $T_{\mathcal{G}}^{\text {ind }}$, and $M$ a small model of $T$ (e.g. of size $|T|$ ). Then

(1) The geometry $\left(N^{*}, \mathrm{cl}^{*}\right)$ of $\mathrm{cl}_{H}$ in $(N, H)$ is a disjoint union of infinite dimensional projective geometries over division rings and/or a trivial geometry.

(2) The geometry of the closure operator $\mathrm{cl}$ in $M$ is a disjoint union of subgeometries of projective geometries over division rings.

Proof. (1) Follows by Lemmas 8.5, 8.6 and 8.8

(2) By Lemma 2.6. any structure of the form $(M, H)$ where $M \models T$, and $H(M)$ is an independent set of generics, can be embedded, in an $H$-independent way, in a sufficiently saturated $H$-structure. Thus we may assume that $(M, \emptyset) \subset(N, H)$ with $M \downarrow_{\emptyset} H(N)$. Then cl-independence over $\emptyset$ in $M$ coincides with cl-independence in $N$ over $H(N)$, i.e. $\mathrm{cl}_{H}$-independence. Thus, we have a natural embedding of the associated geometry of $(M, \mathrm{cl})$ into $\left(N^{*}, \mathrm{cl}^{*}\right)$. The result now follows by $(1)$.

Remark 8.10. The previous proposition also holds with the weaker assumption that the pregeometry $\left(N, \mathrm{cl}_{H}\right)$ is modular instead of asking that $T$ is one-based. All the proofs depend on the properties of the closure operator, not the properties of forking in the full structure.

\section{REFERENCES}

[1] J. Baldwin and M. Benedikt, Stability theory, permutations of indiscernibles, and embedded finite models, Trans. Amer. Math. Soc. 352 (2000), 4937-4969.

[2] I. Ben Yaacov, A. Pillay, and E. Vassiliev, Lovely pairs of models, Ann. Pure Appl. Logic 122 (2003), no. 1-3, 235-261.

[3] A. Berenstein and J. Kim, Dense codense predicates and the NTP2, to be published in Math. Log. Quart.

[4] A. Berenstein and E. Vassiliev, On lovely pairs of geometric structures, Annals of Pure and Applied Logic, Volume 161, Issue 7, April 2010, pp. 866-878.

[5] A. Berenstein and E. Vassiliev, Weakly one-based geometric theories, Journal of Symbolic Logic, v. 77, no. 2, June 2012.

[6] A. Berenstein and E. Vassiliev, Geometric structures with a dense independent subset, Selecta Mathematica, January 2016, Volume 22, Issue 1, pp 191-225.

[7] G Boxall, Lovely pairs and dense pairs of real closed fields, PhD thesis, University of Leeds.

[8] J. Carmona, Forking geometry on theories with an independent predicate, Archive for Mathematical Logic, February 2015, Volume 54, Issue 1-2, pp. 247-255.

[9] E. Casanovas and M. Ziegler, Stable theories with a new predicate, Journal of Symbolic Logic, Vol 66, (2001), pp. 1127-1140.

[10] z. Chatzidakis and A. Pillay, Generic structures and simple theories, Annals of Pure and Applied Logic, Vol 95, 1998, pp 71-92.

[11] A. Dolich, C. Miller and C. Steinhorn, Expansions of O-minimal Structures by Dense Independent Sets, Annals of Pure and Applied Logic, Volume 167, Issue 8, August 2016, Pages 684-706.

[12] A. Fornasiero, Dimension, matroids, and dense pairs of first-order structures, APAL, Volume 162, No. 7, 2011, pp. 514-543.

[13] M. Hall, The theory of groups, Macmillan, New York, 1959.

[14] D. Marker, Model Theory of Difference fields, in Model Theory of Fields, D. Marker, M. Messmer, and A. Pillay, Lecture Notes in Logic, Volume 5 Berlin: Springer-Verlag, 1996.

[15] A. Pillay The geometry of forking and groups of finite Morley rank Journal of Symbolic Logic. 60(4) (1995), pp 1251-1259.

[16] J. Goode Some trivial considerations, Journal of symbolic logic, 56(02) (1991), pp 624-631.

[17] A. Pillay A note on CM-triviality and the geometry of forking, Journal of Symbolic Logic. 65(1) (2000), pp 474-480. 
[18] A. Pillay Lecture notes on strongly minimal sets with a generic automorphism, notes of a graduate course in UIUC, 1999, available at http://www3.nd.edu/ apillay/.

[19] B. Poizat Paires de Structures Stables, Journal of Symbolic Logic, 48 (1983), pp 239-249.

[20] E. Vassiliev, Generic pairs of SU-rank 1 structures, Annals of Pure and Applied Logic, 120, (2003), pp. 103-149.

[21] M. Ziegler, An exposition of Hrushovski's new Strongly Minimal Set, Annals of Pure and Applied Logic 164 (12), (2013), pp 1507-1519.

Universidad de los Andes, Cra 1 No 18A-10, Bogotá, Colombia

$U R L$ : www.matematicas. uniandes.edu.co/ aberenst

Universidad Antonio Nariño, Calle 58 A No. 37 - 94, Bogotá, Colombia

E-mail address: jfcarmonag@gmail.com

Grenfell Campus, Memorial University of Newfoundland, Corner Brook, NL A2H 6P9, Canada

E-mail address: yvasilyev@grenfell.mun.ca 